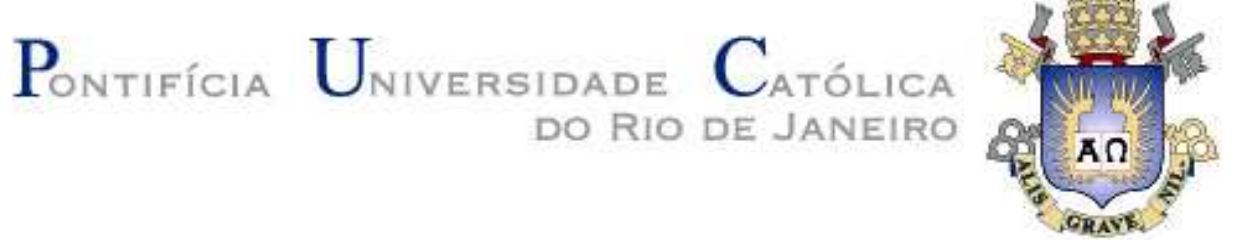

Julien Pierre Castello Branco Jonqua

Modelo de Programação Matemática Estocástica para o Planejamento Estratégico da Cadeia de Petróleo Sob Incerteza

Dissertação de Mestrado (Opção profissional)

Dissertação apresentada como requisito parcial para obtenção do título de Mestre pelo Programa de Pós-Graduação em Engenharia Industrial da PUC-Rio.

Orientador: Prof. Silvio Hamacher

Rio de Janeiro Novembro de 2012 
Julien Pierre Castello Branco Jonqua

\section{Modelo de Programação Matemática Estocástica para o Planejamento Estratégico da Cadeia de Petróleo Sob Incerteza}

Dissertação apresentada como requisito parcial para obtenção do título de Mestre (opção profissional) pelo Programa de Pós-Graduação em Engenharia Industrial da PUC-Rio. Aprovada pela Comissão Examinadora abaixo assinada.

Prof. Silvio Hamacher

Orientador e Presidente

Departamento de Engenharia Industrial - PUC-Rio

Prof. Fabrício Carlos $\mathbf{P}$ de Oliveira Departamento de Engenharia Industrial - PUC-Rio

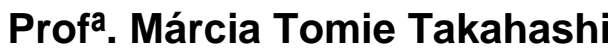

Petróleo Brasileiro - Rio de Janeiro

Prof. José Eugênio Leal Coordenador Setorial do Centro Técnico Científico - PUC-Rio 
Todos os direitos reservados. É proibida a reprodução total ou parcial do trabalho sem autorização da universidade, do autor e do orientador.

\section{Julien Pierre Castello Branco Jonqua}

Graduou-se em Engenharia de Elétrica, com ênfase em Sistemas de Apoio à Decisão na Puc-Rio em 2007. Trabalhou como analista de fundos de investimento na Quantum, de 2007 a 2008. Em 2008, teve uma breve passagem pelo Banco BBM, onde atuou como analista estratégico, na área de Back-Office de fundos offshore. Ainda em 2008, ingressou na Petrobras no cargo de Analista de Pesquisa Operacional, onde trabalha até hoje, desenvolvendo modelos de otimização e provendo soluções de pesquisa operacional para as mais diversas áreas da Companhia.

\footnotetext{
Jonqua, Julien Pierre Castello Branco

Modelo de programação matemática estocástica para o planejamento estratégico da cadeia de petróleo sob incerteza / Julien Pierre Castello Branco Jonqua ; orientador: Silvio Hamacher. - 2012.

59 f. : il. (color.) ; $30 \mathrm{~cm}$

Dissertação (mestrado)-Pontifícia Universidade Católica do Rio de Janeiro, Departamento de Engenharia Industrial, 2012.

Inclui bibliografia.

1. Engenharia industrial - Teses. 2. Otimização sob incerteza. 3. Cadeia integrada do petróleo. 4. Programação estocástica. 5. Decomposição. I. Hamacher, Silvio. II. Pontifícia Universidade Católica do Rio de Janeiro. Departamento de Engenharia Industrial. III. Título.
} 
A minha linda esposa Natasha, fonte de inspiração e ternura, você faz de mim uma pessoa melhor. A minha Família sensacional, orgulho de fazer parte! 


\section{Agradecimentos}

Ao orientador Silvio Hamacher, pela excelente orientação e pelos ensinamentos e aprimoramentos acadêmicos;

Ao co-orientador e amigo Sergio Bruno, pela participação efetiva em todas as fases desta dissertação;

Ao gerente Roberto Iachan pelo incentivo e flexibilidade concedida para a realização do mestrado;

Aos doutores Fabrício Oliveira e Leonardo Lustosa, e à doutora e colega Marcia Takahashi, pela pronta disposição de participar da banca examinadora;

Aos amigos Leonardo Moraes e Luiz Carlos Sousa, pelos comentários, sugestões e ajuda dispensada ao longo deste trabalho;

Aos meus queridos tios David e Lella Harris, por todo investimento feito no meu desenvolvimento e, principalmente, pelo amor incondicional;

À Petróleo Brasileiro S.A. pela oportunidade concedida e excelente política de investimento na capacitação de seus funcionários. 


\section{Resumo}

Jonqua, Julien Pierre; Hamacher, Silvio. Modelo de Programação Matemática Estocástica para o Planejamento Estratégico da Cadeia de Petróleo Sob Incerteza. Rio de Janeiro, 2012. 59p. Dissertação de Mestrado (Opção profissional) - Departamento de Engenharia Industrial, Pontifícia Universidade Católica do Rio de Janeiro.

O presente trabalho tem como foco o estudo do Sistema Petrobras, no que tange o planejamento estratégico dos investimentos da Companhia, sob a ótica da cadeia integrada do petróleo. A partir de um dos modelos matemáticos mais utilizados (e há mais tempo) na empresa, diversas decisões estratégicas de suma importância são suportadas, de modo a maximizar seu resultado operacional ao longo de um horizonte de tempo da ordem de 10 (dez) anos. Com embasamento na literatura atual, evoluções são propostas e testadas no modelo matemático. Primeiramente são introduzidas técnicas de programação estocástica em dois estágios, onde as decisões de investimento são representadas por variáveis de primeiro estágio; e a operação de todo o sistema - desde o refino até a comercialização do petróleo e derivados, passando por toda a questão logística passa a fazer parte do segundo estágio, após a realização / revelação dos parâmetros estocásticos. Em um segundo passo, técnicas de decomposição são aplicadas para contornar eventuais limitações geradas pelo grande porte atingido pelo modelo, que cresce proporcionalmente ao número de cenários envolvidos na otimização. Os resultados mostram que o modelo estocástico começa a esbarrar nestas limitações a partir da resolução de problemas com mais de 30 cenários. Por outro lado, apesar do tempo computacional consideravelmente maior, o modelo decomposto chegou a resolver até 80 cenários, nos testes realizados.

\section{Palavras-chave}

Otimização Sob Incerteza; Cadeia Integrada do Petróleo; Programação Estocástica; Decomposição 


\section{Abstract}

Jonqua, Julien Pierre; Hamacher, Silvio (Advisor). Stochastic Mathematical Programming Model for Strategic Planning of the Oil Supply Chain Under Uncertainty. Rio de Janeiro, 2012. 59p. M.Sc. Dissertation - Departamento de Engenharia Industrial, Pontifícia Universidade Católica do Rio de Janeiro.

This work focuses on the study of Petrobras, regarding the strategic planning of the Company's investments, from an integrated oil supply chain perspective. From one of the most widely used mathematical models in the Company, several strategic decisions of great importance are supported, so as to maximize its operating result over a time horizon of approximately 10 (ten) years. Based in current literature, developments are proposed and tested in the mathematical model. First, two-stage stochastic programming techniques are introduced, where investment decisions are represented by first-stage variables; and system's operation - from oil refining and sales to the entire logistics issue by second-stage variables, after realization of the stochastic parameters. In a second step, decomposition techniques are applied to circumvent any large scale limitations. The results show that the stochastic model starts to reach these limitations in problems with 30 scenarios or more. On the other hand, despite the considerably greater computational time, the decomposed model was able to solve up to 80 -scenarios problems, during the tests.

\section{Keywords}

Optimization Under Uncertainty; Oil Supply Chain; Stochastic Programming; Decomposition 


\section{Sumário}

1 Introdução 11

1.1. Sistema Petrobras $\quad 11$

1.2. Estrutura do trabalho 13

2 Revisão Bibliográfica $\quad 15$

2.1. Cadeira Integrada do Petróleo 15

2.2. Incorporação de Incertezas $\quad 17$

2.3. Métodos de Decomposição 20

2.3.1. Decomposição de Benders 21

2.3.2. Método L-Shaped 23

2.4. Medidas Comparativas $\quad 26$

3 Metodologia $\quad 28$

3.1. Equacionamento (Modelo Determinístico) 28

3.2. Versão Estocástica 38

3.3. Decomposição Multicortes 40

4 Testes e Resultados 43

4.1. Configuração do ambiente utilizado 43

4.2. Cenário Base 44

4.3. Cenários Estocásticos 45

4.3.1. Seleção de cenários $\quad 45$

4.3.2. Rodadas Estocásticas $\quad 46$

4.3.3. Rodadas Decompostas $\quad 49$

5 Conclusão 53

$\begin{array}{ll}\text { Referências Bibliográficas } & 55\end{array}$ 


\section{Lista de figuras}

Figura 1.1 - Representação esquemática das atividades modeladas no PLANINV 13

Figura 4.1 - Projeção do tempo de otimização 48

Figura 4.2 - Convergência dos bounds para 3 cenários $\quad 50$

Figura 4.3 - Convergência dos bounds para 10 cenários $\quad 51$

Figura 4.4 - Convergência dos bounds para 30 cenários $\quad 51$

Figura 4.5 - Convergência dos bounds para 80 cenários 52

\section{Lista de tabelas}

Tabela 2.1 - Literatura e classificação por foco e abordagem (Senne, 2009) 16

Tabela 2.2 - Literatura e classificação por aplicação e técnica (Leiras et al., 2011) 19

Tabela 3.1 - Conjuntos e suas descrições 28

Tabela 3.2 - Parâmetros e suas descrições 30

Tabela 3.3 - Variáveis e suas descrições 31

Tabela 3.4 - Parâmetros Estocásticos 39

Tabela 4.1 - Estatísticas das rodadas estocásticas 48

Tabela 4.2 - Estatísticas das rodadas estocásticas $\quad 52$ 
Não basta apreciar a beleza de um jardim, é preciso também acreditar que há fadas nele?

Douglas Adams 


\section{Introdução}

1.1.

Sistema Petrobras

Em meados da década de 70, técnicos da Petrobras conceberam um modelo matemático de programação linear, a fim de subsidiar estudos envolvendo o planejamento de investimentos em produção, refino e transporte de petróleo e derivados, do sistema Petrobras.

A partir de então o modelo, chamado de Modelo de Planejamento dos Investimentos (PLANINV), vem sendo constantemente aprimorado, com o intuito de estar sempre próximo da realidade atual e futura da empresa e da indústria do petróleo.

Resumidamente o PLANINV representa as principais atividades da Petrobras no Brasil: desde a produção nas fontes; passando por todo o processo de refino dos diferentes petróleos, transformados nos mais diversos tipos de derivados; considerando toda a logística envolvida, as características das unidades que compõem o parque de refino, além das especificações dos produtos demandados em cada mercado. O modelo também considera as possibilidades de importação / exportação de petróleos e derivados; e potenciais investimentos, seja na ampliação ou criação de unidades de refino, seja na capacidade de transporte de determinados arcos, ou ainda no aumento da produção.

A Petrobras, diferentemente de muitas empresas de petróleo, concentra a maior parte dos seus negócios (refino, produção e venda de óleo, gás natural e derivados) em uma mesma região, no Cone Sul. Grande parcela desta produção nacional é processada nas refinarias e UPGN's (Unidades de Processamento de Gás Natural) desta mesma região, assim como é necessário um complexo sistema logístico para o seu escoamento e atendimento ao mercado. A concentração geográfica da Companhia no Cone Sul e a distância dos grandes centros produtores e consumidores mundiais de petróleo e derivados criam uma proteção econômica e fortalecem ainda mais a atratividade da atuação integrada das áreas de negócio da Empresa. Neste contexto de grande integração das atividades da Petrobras e complexidade do sistema de abastecimento do 
país, muitas vezes é difícil aferir as consequiências isoladas de um projeto de investimento.

A interdependência de projetos implica na existência de externalidades que cada um isoladamente gera sobre os demais, inclusive sobre os já existentes, tornando necessário avaliar a influência de um projeto em estudo sobre a totalidade do sistema do qual ele participa.

O equacionamento do modelo busca então representar a integração da oferta de petróleo - dos campos de petróleo nacionais e possibilidades de importação e de exportação de petróleo e derivados - com o parque de refino, abrangendo toda a logística de transporte de petróleos e derivados, e sua inter-relação com a demanda de derivados do país.

Como resposta, o PLANINV determina a forma otimizada de operar o Sistema Petrobras, respeitando todas as interdependências entre áreas de negócios e os limites operacionais, viabilizando a maximização do seu resultado operacional em um horizonte de tempo definido.

Sua escala de tempo é anual, e uma corrida típica pode cobrir um período de cerca de 10 anos. Alguns dos dados de entrada necessários para o modelo são: a curva de produção de óleo do E\&P (Área de Exploração e Produção da Cia); a demanda do mercado nacional (volumes, especificações e preços de derivados comercializados); as possibilidades de exportação e importação de petróleo e derivados (limites de volumes e preços); o parque de refino disponível (refinarias e suas unidades de processamento); a malha de distribuição disponível (rodoviária, dutoviária, cabotagem, entre outros); as possibilidades de expansão ou implantação de novas refinarias, novas unidades ou novos arcos de transporte (incluindo valor do investimento, aumento possível de capacidade e a data mais provável de implantação).

Diversas estratégias podem resultar da otimização do modelo, com base nos dados de entrada. Estão entre as principais: processar ou exportar os óleos nacionais; alocação de óleo para abastecimento de cada refinaria; produzir ou importar os derivados, a partir da demanda do mercado interno; modal de transporte para petróleo e derivados dentro do país, de forma a garantir o abastecimento de refinarias e mercados consumidores; implantar ou não os novos investimentos em unidades de processamento e facilidades de transporte.

É necessário salientar que alguns custos e receitas que não afetam a otimização, não são considerados na maximização do resultado. $\mathrm{O}$ custo de exploração do óleo 
nacional e a receita da venda de produtos de mercado estabelecido podem ser citados como exemplo. A consequiência disso é que os resultados econômicos do PLANINV devem ser usados apenas de forma comparativa, incremental.

Como pode ser visto no esquema abaixo, as atividades representadas no modelo buscam cobrir todo o segmento de atuação da Companhia:

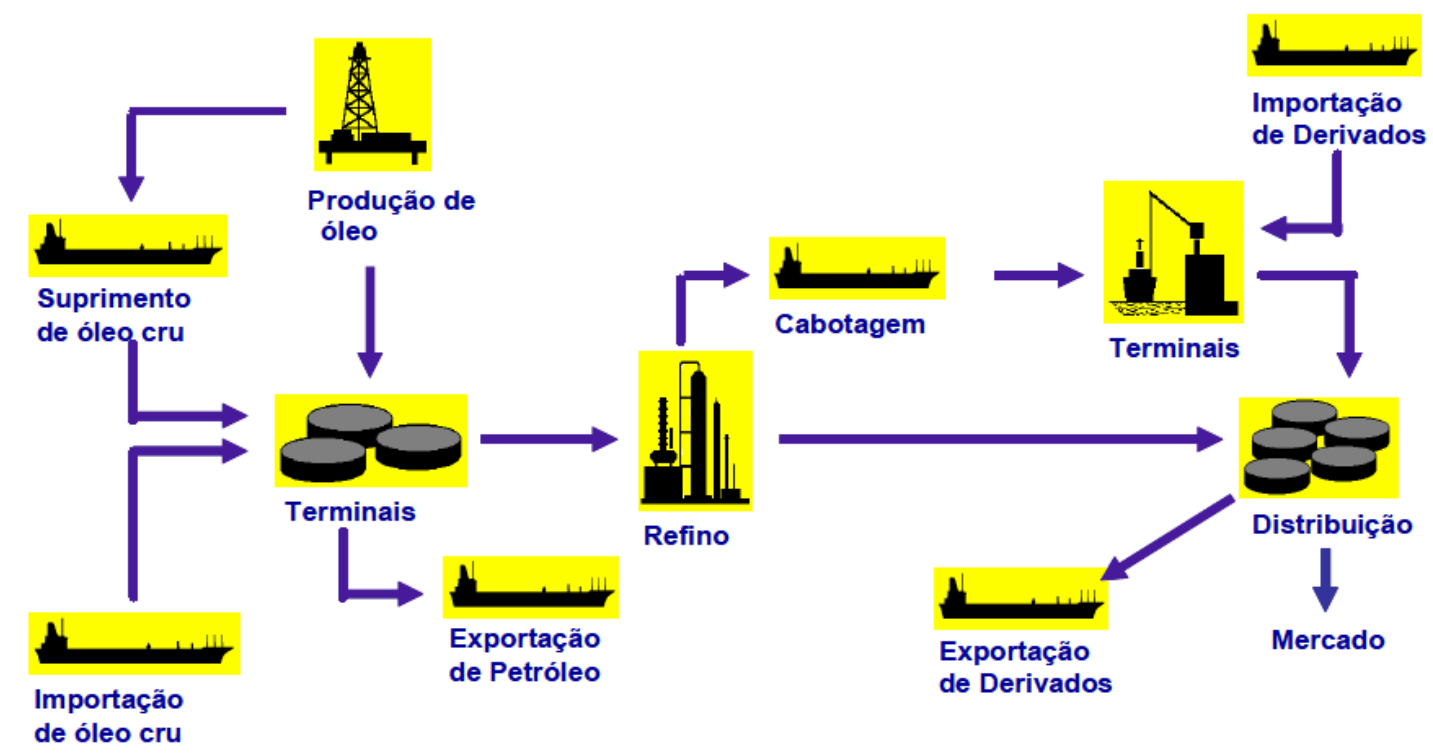

Figura 1.1 - Representação esquemática das atividades modeladas no PLANINV

\section{2.}

\section{Estrutura do Trabalho}

A estrutura da presente dissertação está organizada em cincos capítulos, incluindo este introdutório.

O Capítulo 2 faz uma revisão dos principais trabalhos encontrados na literatura que abordam a cadeia de suprimentos do petróleo, sob a ótica da programação matemática. Inicialmente, o foco se concentra em trabalhos que abordam a cadeia de forma integrada, citando também alguns trabalhos que tratam partes da cadeia separadamente, dada a alta complexidade dos problemas envolvidos. A segunda seção do capítulo é voltada aos trabalhos que consideram a incorporação de incertezas em 
seus modelos. Em seguida, são revisados alguns trabalhos que utilizam métodos de decomposição na estratégia de resolução de modelos estocásticos; e a teoria matemática por trás da decomposição é abordada. Por fim, são apresentadas algumas medidas comparativas utilizadas para valorar uma solução estocástica, ante uma determinística.

No Capítulo 3, é descrita a metodologia utilizada no trabalho atual. Primeiramente, o equacionamento determinístico do modelo PLANINV é descrito, com algumas ressalvas e simplificações pertinentes, dado seu caráter corporativo. Em seguida, é apresentada sua versão estocástica, via identificação dos parâmetros estocásticos, e classificação das variáveis entre primeiro e segundo estágio. Por fim, o processo iterativo utilizado para resolver o modelo via decomposição é detalhado.

Os testes realizados e os principais resultados são apresentados no Capítulo 4, que é dividido em três partes: a primeira descreve a configuração do ambiente de testes; a segunda explicita a construção do Cenário Base e os resultados determinísticos; e a última expõe todos os resultados relativos aos testes com cenários estocásticos, fazendo comparações entre os métodos utilizados na resolução.

Por fim, o Capítulo 5 encerra o trabalho com as conclusões e sugestões de continuação da pesquisa. 


\section{2 \\ Revisão Bibliográfica}

\section{1.}

\section{Cadeira Integrada do Petróleo}

A alta complexidade da cadeia de suprimento do petróleo é freqüentemente citada na literatura, como em Hussain et al. (2006). A maioria dos autores a divide em dois segmentos, upstream e downstream, havendo ainda aqueles que optam pela segmentação em três, incluindo o midstream.

No formato mais comum, como em Manzano (2005) ou Carneiro (2008), por exemplo, o upstream compreende basicamente as atividades de exploração e produção (E\&P) do petróleo; enquanto o downstream abrange toda a parte logística, refino, comercialização e distribuição, de petróleo e derivados.

Mesmo utilizando esta separação, há complexos subproblemas a serem resolvidos em cada segmento da cadeia; o que segundo Jia e Ierapetritou (2003), torna complicado o gerenciamento integrado das atividades, podendo resultar em modelos matemáticos computacionalmente intratáveis.

Neiro e Pinto (2004) constataram, à época, que apenas partes da cadeia de suprimentos do petróleo haviam sido estudadas com um adequado nível de precisão. Segundo Ribas et al. (2008), apesar dos trabalhos publicados modelando suprimento, estoque, refino e distribuição, nenhum deles tratava da cadeia integrada, considerando desde os produtores até as bases primárias e secundárias. Além disso, os modelos de nível estratégico não contemplavam a análise de investimentos em refino e infraestrutura de transporte. Os autores então apresentaram um modelo MILP para o planejamento estratégico da cadeia integrada de petróleo, considerando também as possibilidades de investimento.

Senne (2009) constata que a maioria dos modelos de otimização vistos na literatura lida com partes da cadeia do petróleo independentemente. $\mathrm{O}$ autor apresenta a tabela abaixo, classificando os trabalhos citados por abordagem matemática utilizada, e a parte da cadeia em que focam: 


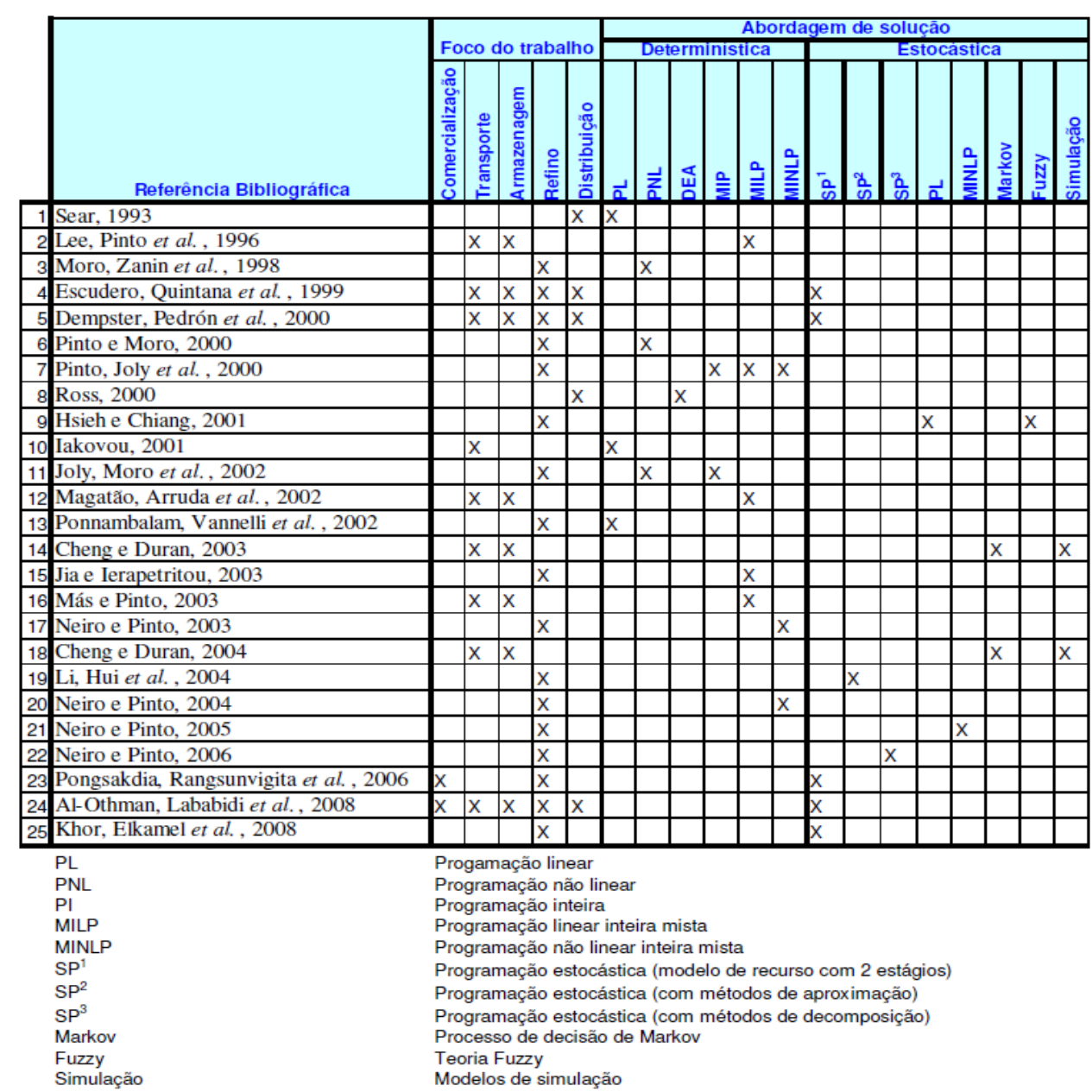

Tabela 2.1 - Literatura e classificação por foco e abordagem (Senne, 2009)

Senne (2009) então propõe a incorporação de incertezas no plano de abastecimento de uma empresa da indústria do petróleo, sob a ótica da cadeia integrada. A técnica utilizada é a de programação estocástica com modelos de recursos de dois e multi-estágio; onde os parâmetros incertos são descritos através de distribuições discretas. No total, são analisados onze modelos estocásticos mais o modelo base determinístico, e são percebidos diferentes graus de impacto para as diversas incertezas.

Já Neiro e Pinto (2003) iniciam a extensão do modelo simples (que considera cada refinaria separadamente) para o modelo corporativo, que abrange diversas refinarias interconectadas. O planejamento integrado de refinarias se mostra claramente vantajoso quando comparado ao modelo que as considera independentemente. Em Neiro e Pinto (2004), o trabalho evolui para um modelo geral do planejamento operacional da cadeia do petróleo. A modelagem abrange unidades de processamento, tanques de estocagem, refinarias, terminais, redes de dutos; além de considerar a complexa topologia que 
interliga os nós. O resultado é um modelo multiperiodal MINLP de grande porte. Três casos são considerados e verifica-se que diferentes estratégias devem ser adotadas, dependendo da amplitude e onde se localizam as incertezas. Com isso, fica ilustrada a necessidade de se coordenar o planejamento da produção, de modo a balancear o comportamento dinâmico da cadeia integrada, face aos diferentes cenários apresentados.

\section{2. \\ Incorporação de Incertezas}

Muitos modelos de programação estocástica são inicialmente formulados como modelos determinísticos. No entanto, se alguns dos parâmetros do modelo determinístico são incertos e este modelo se apresenta sensível a alterações destes parâmetros, então é apropriado considerar programação estocástica para solução desse problema (Higle e Sen, 1999).

Apesar da maioria dos modelos propostos basearem-se em programação determinística, incertezas quanto a preços, demandas e outras condições de mercado podem tornar não robustas, ou mesmo inviáveis, decisões tomadas a partir destes modelos (Li et al., 2004). Lababidi et al. (2008) destacam ainda a existência de incertezas quanto a disponibilidade e qualidade de petróleos, capacidade de processamento e rendimentos dos derivados. Há mais de cinqüenta anos, outros autores (Beale, 1955; Dantzig, 1955) já davam a entender que, devido à existência de incertezas nos parâmetros dos problemas reais, que variam entre o momento da modelagem e o momento em que a decisão deve ser tomada, a modelagem sem a incorporação de incertezas não era a mais adequada para representar a verdadeira dinâmica do mundo real.

Kallrath (2005) apresentou uma visão geral do progresso recente da pesquisa em otimização global, fazendo uma revisão de várias abordagens de técnicas, como programação não-linear inteira mista e outras, usadas em problemas reais ou citadas na literatura. A partir destas abordagens, o autor argumenta que as dificuldades em modelar problemas de otimização sob incerteza são muito maiores do que aquelas encontradas na otimização determinística.

Carneiro et al. (2010) deram continuidade ao trabalho previamente elaborado (Ribas et al., 2008), com a inserção de medidas de risco, considerando-se as incertezas 
relacionadas ao problema. Desta forma, e preenchendo uma lacuna deixada na literatura, os autores propuseram a inclusão de gerenciamento de risco (financeiro), no problema de otimização de portfólio no planejamento da cadeia integrada de petróleo, sob incerteza. Para isso, construíram um modelo de programação estocástica de dois estágios, aplicando o CVaR (Conditional Value at Risk) como medida restritiva de risco.

Os autores Escudero et al. (1999) já haviam utilizado análise de cenários em dois estágios, para abordagem de incertezas nos custos de suprimento, demandas e preços dos produtos. O modelo de programação linear proposto contempla as atividades de logística de suprimento, transformação (refino) e distribuição com o objetivo de atendimento da demanda ao mínimo custo total. As incertezas foram modeladas através de um conjunto de cenários (resultando em um modelo estendido) e as decisões quanto à política de suprimento, transformação e distribuição para os períodos iniciais são tomadas, sem a necessidade de antecipar as decisões relacionadas aos outros períodos.

O problema da escolha de qual petróleo comprar e o quanto produzir de cada derivado é considerado em Pongsakdia et al. (2006); em que novamente foi empregada programação estocástica com modelos de recurso de dois estágios.

Ainda nesta linha, Nascimento (2011) trata a questão do planejamento de uma refinaria a médio prazo, considerando-se a influência de parâmetros incertos nas decisões de produção. Primeiro foi desenvolvido um modelo determinístico linear do sistema estudado, composto por equações de balanceamento de fluxos de material e de especificação de propriedades de produtos finais. Em seguida, foram gerados modelos de programação estocástica, cada um considerando um conjunto de incertezas (na demanda, nos preços e nas capacidades). Por último, dado o porte dos modelos, foi necessária a utilização de um método de decomposição chamado de L-Shaped, para se obter a solução ótima.

O planejamento das atividades em refinarias também foi abordado por Elkamel et al. (2008), em que incertezas quanto preço, demanda e rendimento dos produtos foram consideradas através de modelos de programação estocástica. Em quatro abordagens diferentes, os autores combinaram programação estocástica de dois estágios com recurso fixo, modelo média-variância de Markowitz, e o modelo Desvio Absoluto Médio (MAD) para a minimização do valor esperado e/ou da variância dos valores esperados associados aos parâmetros incertos. 
Leiras et al. (2011) fazem uma revisão da literatura sobre modelos de planejamento de refinarias, enfatizando as principais técnicas utilizadas para lidar com otimização sob incerteza. Os trabalhos são classificados de acordo com três quesitos principais: segmento da cadeia que abordam (upstream, midstream ou downstream), nível de planejamento (estratégico, tático ou operacional), e classificação matemática (quanto à linearidade e presença de variáveis inteiras); conforme tabela abaixo. O objetivo final é identificar lacunas na literatura e potenciais oportunidades de pesquisa na área. Nesse sentido, o trabalho conclui que existe uma grande oportunidade de publicação na área de otimização estocástica robusta e otimização robusta. Além disso, constatou-se uma maior carência de publicação de trabalhos relacionados a planejamento estratégico de refinarias.

\begin{tabular}{|c|c|c|c|c|c|c|c|c|c|c|}
\hline \multirow{2}{*}{ Autor (Ano) } & \multicolumn{3}{|c|}{ Aplicação } & \multicolumn{3}{|c|}{ Nivel de Decisão } & \multicolumn{4}{|c|}{ Técnica de programação } \\
\hline & Up & Mid & Down & Estrat. & Tático & Oper. & LP & NLP & MILP & MINLP \\
\hline Al-Othman et al. (2008) & $\mathrm{x}$ & $\mathrm{x}$ & $\mathrm{x}$ & & $\mathrm{x}$ & & & & $\mathrm{x}$ & \\
\hline Khor et al. (2008) & & $\mathrm{x}$ & & & $\mathrm{x}$ & & $\mathrm{x}$ & & & \\
\hline Lakkahanawat e Bagajewicz (2008) & & $\mathrm{x}$ & & & & $\mathrm{x}$ & $\mathrm{x}$ & & & \\
\hline Li e He et al. (2008) & & $\mathrm{x}$ & & & & $\mathrm{x}$ & $\mathrm{x}$ & $\mathrm{x}$ & & $\mathrm{x}$ \\
\hline Pongsakdi et al . (2006) & & $\mathrm{x}$ & & & & $\mathrm{x}$ & $\mathrm{x}$ & & & \\
\hline Neiro e Pinto (2005) & & $\mathrm{x}$ & & & & $\mathrm{x}$ & & & & $\mathrm{x}$ \\
\hline Lababidi et al. (2004) & & $\mathrm{x}$ & $\mathrm{x}$ & & $\mathrm{x}$ & & & & & $\mathrm{x}$ \\
\hline Li e Hui et al. (2004) & & $\mathrm{x}$ & & & $\mathrm{x}$ & & & & $\mathrm{x}$ & $\mathrm{x}$ \\
\hline Hsieh e Chiang (2001) & & $\mathrm{x}$ & $\mathrm{x}$ & & $\mathrm{x}$ & & $\mathrm{x}$ & & & \\
\hline Dempster et al. (2000) & & $\mathrm{x}$ & $\mathrm{x}$ & & $\mathrm{x}$ & & $\mathrm{x}$ & & & \\
\hline Escudero et al. (1999) & & $\mathrm{x}$ & $\mathrm{x}$ & & $\mathrm{x}$ & & $\mathrm{x}$ & & & \\
\hline Liu e Sahinidis (1996) & & $\mathrm{x}$ & & & $\mathrm{x}$ & & & & $\mathrm{x}$ & \\
\hline
\end{tabular}

Tabela 2.2 - Literatura e classificação por aplicação e técnica (Leiras et al., 2011)

Neiro e Pinto (2005) focam no processo decisório envolvido no planejamento da produção de petróleo. O modelo proposto é baseado em uma formulação de programação não-linear, desenvolvida para o plano de produção de um único período. Primeiro, o modelo incorpora vários períodos de planejamento e seleção de diferentes tipos de petróleo bruto. Em seguida, incertezas relacionadas a preço e demanda (de petróleo e produtos) são consideradas em forma de distribuições discretas de probabilidade. Finalmente, são adicionadas as restrições de movimentação do petróleo bruto. O modelo resultante é um MINLP (Mixed Integer Non-Linear Program), aplicado com sucesso a um caso real da refinaria REVAP da Petrobras. 
Também há bons trabalhos na área de scheduling, com foco no planejamento operacional (segundo classificação utilizada em Leiras et al., 2011): Herroelen e Leus (2005), Joly et al. (2002), Grossmann et al. (2001), Pinto et al. (2000), e Verderame et al. (2010).

\section{3. \\ Métodos de Decomposição}

O mesmo tipo de problema resolvido em Neiro e Pinto (2005) é abordado em Neiro e Pinto (2006), entretanto com uma estratégia de solução diferente. Desta vez os autores aliaram programação estocástica com técnicas de decomposição Lagrangeana para explorar a estrutura bloco-diagonal do problema e, com isso, reduzir o esforço computacional.

Com o mesmo intuito de redução de esforço computacional, Nascimento (2011) aplicou o método L-Shaped de decomposição, cujo algoritmo foi desenvolvido por Slyke e Wets (1969). Basicamente, o algorítmo decompõe o problema original em um problema mestre associado às variáveis de primeiro estágio e em um subproblema associado às variáveis de segundo estágio. Esse método é conhecido em outras áreas da programação matemática como Decomposição de Benders (Benders, 1962), originalmente utilizado para resolver problemas lineares inteiros mistos.

Más e Pinto (2003) também desenvolveram uma estratégia de decomposição baseada na resolução de mais de um modelo MILP. Inicialmente, abordaram problemas de scheduling de óleo cru no curto prazo em uma distribuição complexa, que contém portos, refinarias e estrutura de dutos. O problema foi formulado como um modelo MILP de grande porte em tempo contínuo, baseado em eventos difíceis de resolver simultaneamente.

Este tipo de estratégia consiste, metaforicamente falando, em "dividir para conquistar", e não é novidade na literatura. Como exemplos de trabalhos que lançaram mão de métodos de decomposição, com a finalidade de tornar tratáveis problemas de grande porte, podemos citar ao longo da literatura: Geoffrion e Graves (1974), Magnanti et al. (1986), Franca e Luna (1982), Cordeau et al. (2000), Santoso et al. (2005), Uster e Agrahari (2011), Saharidis et al. (2011), Yang e Lee (2011), Peng e Jirutitijaroen (2010), entre outros. 


\subsection{1.}

Decomposição de Benders

O método de resolução e aproximação de problemas de otimização estocástica, mais conhecido e tradicional, é possivelmente o L-Shaped. Na realidade, o método pode ser visto como uma aplicação da Decomposição de Benders, adaptada à otimização estocástica. Deste modo, faz-se necessária uma breve apresentação da metodologia utilizada em Benders.

Seguindo a linha de Pagnoncelli e Bortolossi (2008), considera-se o seguinte problema de otimização linear:

$$
\begin{array}{|rr|}
\text { VAL }=\min _{\substack{\mathbf{x}, \mathbf{y} \\
\text { sujeito a }}} \mathbf{c}^{T} \mathbf{x}+\mathbf{q}^{T} \mathbf{y} \\
& \mathbf{A x}=\mathbf{b}, \\
& \mathbf{T x}+\mathbf{W} \mathbf{y}=\mathbf{h} \\
\mathbf{x}, \mathbf{y} \geq \mathbf{0}
\end{array}
$$

onde c, q, x e y são vetores em $R^{n}$, h e b são vetores de $R^{m}$ e A, T e W são matrizes m $\times$ n. O primeiro passo é eliminar a variável y da formulação do problema, criando um subproblema parametrizado por x. Para isso, a Decomposição de Benders reescreve o problema na forma:

$$
\begin{array}{rr}
\text { VAL }=\min _{\mathbf{x}} & \mathrm{c}^{T} \mathrm{x}+\mathcal{Q}(\mathrm{x}) \\
\text { sujeito a } & \mathbf{A x}=\mathbf{b}, \\
& \mathrm{x} \geq \mathbf{0}
\end{array}
$$

onde $\mathrm{Q}(\mathrm{x})$ é o valor ótimo do subproblema:

$$
\begin{array}{rcc}
\mathcal{Q}(\mathrm{x})=\min _{\mathbf{y}} & \mathbf{q}^{T} \mathbf{y} \\
& \text { sujeito a } & \mathbf{W y}=\mathbf{h}-\mathbf{T x}, \\
& \mathbf{y} \geq \mathbf{0} .
\end{array}
$$

Dualizando (2.3), temos o problema (2.4) definido a seguir:

$$
\begin{aligned}
& \mathcal{Q}^{\prime}(x)=\max _{\mathbf{p}} \quad \mathbf{p}^{T}(\mathbf{h}-\mathbf{T x}) \\
& \text { sujeito a } \quad \mathrm{W}^{T} \mathrm{p} \leq \mathrm{q} \text {. }
\end{aligned}
$$


A dualidade tira a dependência do conjunto admissível $D$ de (2.4) em relação a x, ou seja, para qualquer escolha de x o conjunto admissível de (2.4) é o mesmo:

$$
\mathcal{D}=\left\{\mathrm{p} \in \mathbb{R}^{m} \mid \mathbf{W}^{T} \mathrm{p} \leq \mathrm{q}\right\}
$$

Vamos assumir que o conjunto $D$ é não vazio e denotar seus pontos extremos por $p^{1}, \ldots, p^{I}$ e seus raios extremos por $r^{1}, \ldots, r^{J}$. O problema (2.4) dá origem a duas situações distintas:

- Se $\mathrm{Q}^{\prime}(\mathrm{x})=+\infty$, ou seja, ilimitado, então o simplex devolve o raio extremo $\bar{r}$ de $D$. Em particular $(\bar{r})^{T}(h-T x)>0$.

- Se $\mathrm{Q}^{\prime}(\mathrm{x})<+\infty$, então o simplex devolve um ponto extremo $\bar{p}$ de $D$.

Podemos reescrever o problema (2.4) como:

$$
\begin{array}{|cc|}
\hline \min _{z} & z \\
\text { sujeito a } & \left(\overline{\mathbf{p}}^{i}\right)^{T}(\mathbf{h}-\mathbf{T x}) \leq z, i=1, \ldots, I, \\
& \left(\overline{\mathbf{r}}^{j}\right)^{T}(\mathbf{h}-\mathbf{T x}) \leq 0, j=1, \ldots, J
\end{array}
$$

Não é difícil ver que esse problema possui o mesmo valor ótimo de (2.4). As primeiras I restrições representam os valores da função objetivo de (2.4) avaliada nos pontos extremos de $D$. Como queremos minimizar $z$, o valor ótimo de (2.6) corresponde ao maior valor de $\left(\bar{p}^{i}\right)^{T}(h-T x)$, que é exatamente o ponto ótimo de (2.4). As $\mathbf{J}$ restrições restantes servem para garantir que estamos na situação onde o problema (2.4) é limitado.

Finalmente, usando (2.6), chegamos em uma reformulação do problema original (2.1), conhecida por problema mestre completo (PMC), que é a base para a Decomposição de Benders:

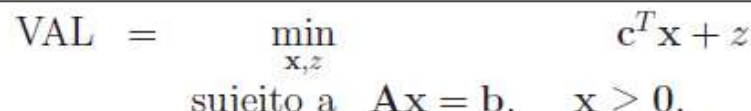

$$
\begin{aligned}
& \left(\mathbf{p}^{i}\right)^{T}(\mathbf{h}-\mathbf{T x}) \leq z, i=1, \ldots, I(*), \\
& \left(\mathbf{r}^{j}\right)^{T}(\mathbf{h}-\mathbf{T x}) \leq 0, j=1, \ldots, J(*) .
\end{aligned}
$$


Essa formulação, apesar de equivalente a (2.1), possui várias diferenças que serão exploradas pela Decomposição de Benders. Primeiramente, a variável y não aparece no PMC. Em seu lugar surge a variável unidimensional z. Além disso, nessa formulação é preciso conhecer os pontos e raios extremos do conjunto $D$. Possivelmente o número de restrições de (2.7) é gigantesco se comparado a (2.1): se o conjunto admissível $D$ for muito facetado, então teremos uma quantidade muito grande de pontos extremos. Aliás, em geral, não temos nem os pontos nem os raios extremos do conjunto $D$ de imediato. A idéia do algoritmo da Decomposição de Benders é considerar um problema semelhante a (2.7) nas etapas intermediárias do algoritmo e ir acrescentando restrições do tipo (*) ao problema em cada passo. Mais precisamente, no passo k do algoritmo, o problema mestre restrito de ordem $\mathrm{k}\left(P M R^{k}\right)$ com apenas $\mathrm{k}$ restrições do tipo (*) é:

$$
\begin{aligned}
\mathrm{VAL}^{k}=\min _{\mathbf{x}, z} & \mathbf{c}^{T} \mathbf{x}+z \\
\text { sujeito a } & \mathbf{A x}=\mathbf{b}, \mathbf{x} \geq \mathbf{0} \\
& \left(\mathbf{p}^{i}\right)^{T}(\mathbf{h}-\mathbf{T x}) \leq z, i=1, \ldots, k-l, \\
& \left(\mathbf{r}^{j}\right)^{T}(\mathbf{h}-\mathbf{T x}) \leq 0, j=1, \ldots l .
\end{aligned}
$$

Para obter pontos e raios extremos de $D$ temos que resolver o problema (2.4) em cada passo do algoritmo. Se ele for finito, então ganhamos um ponto extremo e, se for ilimitado, ganhamos um raio extremo. Eles serão adicionados ao problema $P M R^{k}$, originando o problema $P M R^{k+1}$.

\subsection{2.}

\section{Método L-Shaped}

Considere agora, um problema de otimização estocástica de dois estágios:

$$
\min _{\mathbf{x} \in \mathbf{X}}\left\{\mathrm{cx}+\mathbb{E}\left[\min _{\mathbf{y} \in \mathbf{Y}}\{\mathbf{q}(\omega) \mathbf{y} \mid \mathbf{W}(\omega) \mathbf{y} \sim \mathbf{h}(\omega)-\mathbf{T}(\omega) \mathbf{x}\}\right] \mid \mathrm{Ax}=\mathbf{b}\right\}
$$

O método L-Shaped consiste, em usar a Decomposição de Benders na forma estendida de um problema estocástico de dois estágios. O primeiro passo é reescrever o modelo de recurso em dois estágios (2.9), de maneira análoga ao que foi feito para (2.1). 
Denotando por $\Omega=\left\{\omega^{1}, \omega^{2}, \ldots, \omega^{S}\right\}$ o espaço amostral da variável aleatória subjacente a (2.9) e definindo $p_{s}=\mathrm{P}\left(\omega^{s}\right)$, temos:

$$
\begin{array}{cc}
\mathrm{VAL}=\min _{\mathbf{x}} & \mathbf{c}^{T} \mathbf{x}+\sum_{s=1}^{S} p_{s} Q^{s}\left(\mathbf{x}, \omega^{\mathbf{s}}\right) \\
\text { sujeito a } & \mathbf{A x}=\mathbf{b} \\
& \mathbf{x} \geq \mathbf{0}
\end{array}
$$

onde,

$$
\begin{array}{cc}
Q^{s}\left(\mathbf{x}, \omega^{s}\right)=\min _{\mathbf{y}} & \mathbf{q}^{T} \mathbf{y}^{s} \\
\text { sujeito a } & \mathbf{W y}^{s}=\mathbf{h}^{s}-\mathbf{T}^{s} \mathbf{x} \\
& \mathbf{y}^{s} \geq \mathbf{0}
\end{array}
$$

para cada s de 1 a $\mathrm{S}$. Repare que em (2.11) a matriz W aparece fixa. Esta é uma simplificação para o caso particular com recurso fixo.

Dando seqüência a este caso particular, podemos escrever o problema em sua forma estendida:

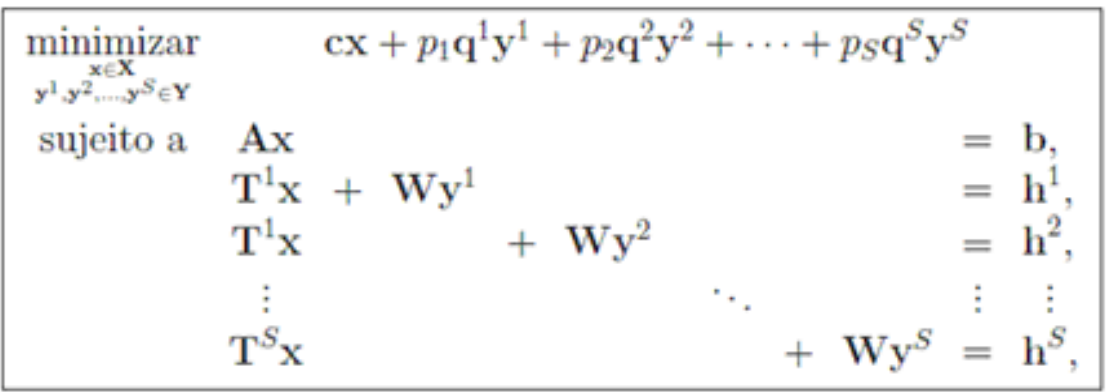

Repare que o problema (2.1) tem o mesmo formato de (2.12), porém com menos restrições. A estrutura da formulação estendida (2.12) naturalmente desacopla os $\mathrm{s}$ problemas (2.11). Dualizando cada um deles, tem-se:

$$
\begin{array}{cc}
Q^{s^{\mathbf{s}}}\left(\mathrm{x}, \omega^{s}\right)=\max _{\substack{\mathbf{p} \\
\text { sujeito a }}} & \mathrm{p}^{T}\left(\mathbf{h}^{s}-\mathrm{T}^{s} \mathrm{x}\right) \\
& \mathbf{W} \mathbf{p} \leq \mathbf{q}^{s} .
\end{array}
$$

Reescrevendo o problema (2.13) da mesma forma que foi feito para (2.6): 


$$
\begin{array}{|cc|}
\hline \min _{z_{s}} & z_{s} \\
\text { sujeito a } & \left(\mathbf{p}_{s}^{i(s)}\right)^{T}\left(\mathbf{h}^{s}-\mathrm{Tx}\right) \leq z_{s}, i(s)=1, \ldots, I_{s}, \\
& \left(\mathrm{r}_{s}^{j(s)}\right)^{T}\left(\mathbf{h}^{s}-\mathrm{Tx}\right) \leq 0, j(s)=1, \ldots, J_{s},
\end{array}
$$

onde $I^{s}$ é o número de pontos extremos do conjunto $D^{s}=\left\{p \mid W p \leq q^{s}\right\}, J^{s}$ é o número de raios extremos de $D^{s}, p_{s}^{i(s)}$ são os pontos extremos de $D$ e $r_{s}^{j(s)}$ são os raios extremos, para cada $s=1, \ldots, S$. Portanto, podemos reescrever o problema na forma estendida como:

$$
\begin{aligned}
& \begin{array}{cc}
\mathrm{VAL}= & \mathbf{c x}+\sum_{\substack{\mathbf{x}, z \\
\text { sujeito a }}}^{S} p_{s} z_{s} \\
& \left(\mathbf{p}_{s}^{i(s)}\right)^{T}\left(\mathbf{h}^{s}-\mathbf{T x}\right) \leq z_{s}, i(s)=1, \ldots, I_{s}, s=1, \ldots, S,
\end{array} \\
& \left(\mathbf{r}_{s}^{j(s)}\right)^{T}\left(\mathbf{h}^{s}-\mathbf{T x}\right) \leq 0, j(s)=1, \ldots, J_{s}, s=1, \ldots, S,
\end{aligned}
$$

que é também chamado de PMC. A partir daí o algoritmo L-Shaped é análogo a Benders. Considera-se o problema mestre restrito $P M R^{k}$ e, em cada passo do algoritmo, adicionam-se os cortes.

Basicamente, o método L-Shaped pode ser apresentado na sua versão clássica ou ainda na versão multicortes. A grande diferença é que no caso multicortes serão acrescentados até S cortes em cada passo, oriundos de cada um dos S problemas (2.13). Além disso, a condição de otimalidade tem que se verificar para todo s, ou seja, $Q\left(\bar{x}^{s}\right)=\bar{z}^{s}$ para s de 1 a S.

Na versão clássica do algoritmo, assumindo por simplicidade que os s problemas (2.13) possuem solução ótima, ao invés de se construir S cortes a partir dessas soluções, constrói-se apenas um corte, que é uma combinação linear convexa dos cortes obtidos. Naturalmente temos apenas uma variável z neste caso, por ter sido gerado apenas um corte:

$$
\sum_{s=1}^{S} p_{s}\left(\mathbf{p}_{s}^{i(s)}\right)^{T}\left(\mathbf{h}_{s}-T_{s} \mathbf{x}\right) \leq z, i(s) \in\left\{1, \ldots, I_{s}\right\}, s=1, \ldots, S .
$$

O algoritmo utilizado na presente dissertação está comentado em detalhes no Capítulo 3. 


\section{4. \\ Medidas Comparativas}

Para fins de comparação das soluções estocásticas com as determinísticas, no capítulo 4, são utilizadas as definiç̧ões apresentadas por Birge e Louveaux (1997).

A solução chamada de espere-e-veja (wait-and-see - WS) corresponde ao valor ótimo do problema quando as realizações futuras dos parâmetros estocásticos são conhecidas, isto é, o tomador de decisão pode esperar e ver o futuro antes de decidir. $\mathrm{Na}$ prática, a solução WS é calculada a partir da otimização determinística de cada um dos cenários individualmente, ponderando-se pela probabilidade de ocorrência do respectivo cenário.

Já a solução aqui-e-agora (here-and-now) correspondente ao problema de recurso (RP) de dois estágios definido neste capítulo, como (2.10). A solução RP é definida como aqui-e-agora, pois a solução de primeiro estágio é decidida sem que se conheçam as realizações futuras dos parâmetros estocásticos, isto é, a decisão é tomada no momento presente sem nenhum conhecimento sobre o futuro.

Com essas duas definições, é possível também definir o valor esperado da informação perfeita (Expected Value of Perfect Information - EVPI), como sendo a diferença entre elas: EVPI $=$ WS - RP.

O EVPI então representa a diferença entre a solução obtida pelo agente com poder de predição perfeita (conhece os eventos futuros) e o agente que resolve o problema sob a hipótese de conhecer apenas a distribuição de probabilidade dos cenários.

Precificar o valor da informação perfeita pode não ser a medida de comparação mais adequada, dado que a solução WS é impossível de ser realizada na prática. Desta forma, também foi apresentada a definição para o valor da solução estocástica (Value of the Stochastic Solution - VSS).

Para calcular o VSS, no entanto, também é necessário apresentar o EEV. O EEV é obtido fixando-se as variáveis de primeiro estágio, com os valores obtidos na solução determinística do Cenário Base, para resolver o problema estocástico com os demais cenários. Na prática, a configuração ótima das variáveis de primeiro estágio obtida na solução do Cenário Base (ou médio), é fixada e utilizada para resolver todos os cenários. A solução de cada um é ponderada por sua respectiva probabilidade de ocorrência. 
O VSS então representa a diferença entre a solução RP e a solução EEV: VSS = RP - EEV; sendo assim, mede o ganho do decisor em ter um modelo estocástico, ou até quanto se pagaria para ter um modelo estocástico. 


\section{3 \\ Metodologia}

O capítulo atual apresenta o equacionamento, com certas simplificações necessárias para preservar o sigilo corporativo, do modelo PLANINV. As modificações propostas nesta dissertação são detalhadas: variáveis são classificadas entre primeiro e segundo estágio, os parâmetros estocásticos são identificados, e por fim é apresentada a metodologia de decomposição utilizada.

\section{1. Equacionamento (Modelo Determinístico)}

Conforme mencionado, a modelagem a seguir apresenta algumas simplificações em relação ao modelo computacional, tanto para preservar o sigilo de um produto corporativo, quanto para facilitar o entendimento geral do problema. A princípio é exposta a versão determinística linear da modelagem. Posteriormente, os parâmetros estocásticos são identificados, e as variáveis são classificadas entre primeiro e segundo estágio.

Desta forma, a versão estocástica da modelagem pode ser considerada uma extensão do problema determinístico. A grosso modo, cada restrição que possua parâmetros estocásticos / variáveis de segundo estágio, passará a ser indexada por cenário na versão estocástica.

Os conjuntos utilizados na modelagem estão descritos na tabela abaixo:

\begin{tabular}{|c|l|c|l|}
\multicolumn{5}{|c|}{ Conjuntos } \\
\hline Índice & \multicolumn{1}{|c|}{ Descrição } & Índice & \multicolumn{1}{c|}{ Descrição } \\
\hline \hline ANO, ANA & Anos do horizonte de estudo & PET, PTL & Petróleos \\
\hline ARC, ARK & Arcos de transporte & POR & Portos (no exterior) com oferta de petróleo \\
\hline \hline BAS & Bases de Consumo & PRD, PRT & Produtos \\
\hline \hline CAM & Campanhas das unidades de processamento & PRJ & Projetos de produção de petróleo / produto \\
\hline \hline FAI & Faixas de sobre-estadia & REF & Refinarias \\
\hline \hline FON & Fontes produtoras de petróleo / produto & SEN & Sentidos de arcos \\
\hline \hline FRT & Frentes (no exterior) de oferta/demanda de produto & TER & Terminais \\
\hline \hline J & Faixas de preço & UNI & Unidades de Processamento \\
\hline
\end{tabular}

Tabela 3.1 - Conjuntos e suas descrições 
Os parâmetros do modelo estão detalhados a seguir:

\begin{tabular}{|c|c|c|}
\hline \multicolumn{3}{|c|}{ Parâmetros } \\
\hline Abreviatura & Descrição & Unidade \\
\hline capad $_{\text {arc }}$ & Volume máximo de ampliação da capacidade do ARCO & M m3 \\
\hline capad $2_{\text {uni }}$ & Vazão da ampliação da carga da UNIDADE & $\mathrm{M} \mathrm{m} 3 / \mathrm{d}$ \\
\hline capat $_{\text {arc }}$ & Volume máximo transferível no ARCO & M m3 \\
\hline $\operatorname{capmax}_{\text {uni }}$ & Vazão máxima processsada na UNIDADE & $\mathrm{M} \mathrm{m} 3 / \mathrm{d}$ \\
\hline capmin $_{\text {uni }}$ & Vazão mínima processsada na UNIDADE & $\mathrm{M} \mathrm{m} 3 / \mathrm{d}$ \\
\hline curvaprod $_{p r j, a n o}^{\text {prd }}$ & Quantidade do PRODUTO produzido pelo PROJETO no PERÍODO & $\mathrm{M} \mathrm{m} 3$ ou M t \\
\hline curvapet $_{p r j, a n o}$ & $\begin{array}{l}\text { Volume de petróleo produzido no PERÍODO posterior ao início do } \\
\text { PROJETO }\end{array}$ & M m3 \\
\hline custo $_{\text {fai,ano }}^{\text {ter }}$ & $\begin{array}{l}\text { Valor corrente do custo de sobrestadia no TERMINAL na } \\
\text { FAIXASOBREESTADIA no ANO }\end{array}$ & M US $\$ / m 3$ \\
\hline custoamp $_{\text {uni }}$ & Valor do custo operacional fixo da ampliação da carga da UNIDADE & MM US\$ \\
\hline custoinv $_{\text {arc,ano }}$ & Valor corrente do custo de implantação/ampliação do ARCO no ANO & MM US\$ \\
\hline custoinv $2_{\text {prj,ano }}$ & Valor corrente do custo do PROJETO no ANO & MM US\$ \\
\hline custoinvca rga $a_{\text {uni,ano }}$ & $\begin{array}{l}\text { Valor corrente do custo de implantação/ampliação da capacidade de carga } \\
\text { da UNIDADE no ANO }\end{array}$ & MM US\$ \\
\hline custoop $_{\text {prj,ano }}$ & $\begin{array}{l}\text { Valor corrente do custo operacional no PERÍODO anterior/posterior ao } \\
\text { início do PROJETO }\end{array}$ & MM US\$ \\
\hline custoop $2_{\text {arc,sen,ano }}$ & Valor corrente do custo operacional do ARCO no SENTIDO no ANO & MM US\$ \\
\hline custoop3 $3_{\text {prd ,uni }}$ & $\begin{array}{l}\text { Valor corrente do custo operacional do processamento do PRODUTO na } \\
\text { UNIDADE }\end{array}$ & M US\$/m3 \\
\hline demanda ${ }_{\text {frt,ano }}^{j, p e t}$ & $\begin{array}{l}\text { Volume máximo de exportação do ÓLEO para a FRENTE na FAIXA de } \\
\text { preço no ANO }\end{array}$ & M m3 \\
\hline demanda $2_{\text {frt,ano }}^{j, p r d}$ & $\begin{array}{l}\text { Quantidade máxima de exportação do PRODUTO para a FRENTE na } \\
\text { FAIXA de preço no ANO }\end{array}$ & $\mathrm{M} \mathrm{m} 3$ ou M t \\
\hline fatinv $v_{\text {arc }, \text { sen }}$ & $\begin{array}{l}\text { Fator multiplicativo da capacidade do ARCO quando operando no sentido } \\
\text { inverso (no sentido normal é 1) }\end{array}$ & Fração \\
\hline fat $_{\text {pet,arc }}$ & Fator multiplicativo do fluxo do ÓLEO no ARCO & Fração \\
\hline fat $2_{\text {ptl,ano }}^{\text {pet }}$ & Proporção do PETRÓLEO componente no PETRÓLEO mistura no ANO & Fração \\
\hline fat3 ${ }_{\text {prd,uni }}$ & Fator multiplicativo da carga do PRODUTO na UNIDADE & Fração \\
\hline
\end{tabular}




\begin{tabular}{|c|c|c|}
\hline \multicolumn{3}{|c|}{ Parâmetros } \\
\hline Abreviatura & Descrição & Unidade \\
\hline fatdesc $_{\text {ano }}$ & Fator de desconto no ANO & Fração \\
\hline fatop $_{\text {uni,ano }}$ & Fator operacional de utilização da UNIDADE no ANO (fut) & Fração \\
\hline $\max _{\text {fai,ano }}^{\text {ter }}$ & $\begin{array}{l}\text { Volume máximo de petróleo e derivados movimentado no TERMINAL na } \\
\text { FAIXASOBRESTADIA no ANO }\end{array}$ & M m3 \\
\hline $\operatorname{mermax}_{b a s, a n o}^{\text {prd }}$ & Quantidade máxima de demanda do PRODUTO na BASE no ANO & M m3 ou M t \\
\hline mermin $_{\text {bas,ano }}^{\text {prd }}$ & Quantidade mínima de demanda do PRODUTO na BASE no ANO & M m3 ou M t \\
\hline $\min _{\text {ref,ano }}^{\text {pet }}$ & Volume mínimo do ÓLEO requerido na REFINARIA no ANO & M m3 \\
\hline oferta ${ }_{\text {por,ano }}^{\text {j,pet }}$ & Volume máximo disponível do ÓLEO no PORTO na faixa J no ANO & M m3 \\
\hline oferta $2_{\text {por,ano }}^{\text {j,prd }}$ & $\begin{array}{l}\text { Quantidade máxima de importação do PRODUTO da FRENTE na faixa J } \\
\text { no ANO }\end{array}$ & M m3 ou M t \\
\hline precoexp frt,ano & Preço corrente de exportação do ÓLEO para a FRENTE na faixa 1 no ANO & M US\$/m3 \\
\hline precoexp $2_{\text {frt,ano }}^{\text {j,prd }}$ & $\begin{array}{l}\text { Preço corrente de exportação do PRODUTO para a FRENTE na faixa } 1 \text { no } \\
\text { ANO }\end{array}$ & $\begin{array}{c}\text { M US\$/m3 ou M } \\
\text { US\$/t }\end{array}$ \\
\hline precoimp frt,ano $_{\text {j,pet }}$ & Preço corrente do ÓLEO no PORTO na faixa1 no ANO & M US\$/m3 \\
\hline precoimp2 $2_{\text {por,ano }}^{\text {j,prd }}$ & $\begin{array}{l}\text { Preço corrente de importação do PRODUTO da FRENTE na faixa } 1 \text { no } \\
\text { ANO }\end{array}$ & $\begin{array}{c}\text { M US\$/m3 ou M } \\
\text { US\$/t }\end{array}$ \\
\hline prodat $_{\text {fon, ano }}^{\text {pet }}$ & Volume do ÓLEO produzido pela FONTE no ANO & M m3 \\
\hline prodat $2_{\text {fon,ano }}^{\text {prd }}$ & Quantidade do PRODUTO produzida pela FONTE no ANO & M m3 ou M t \\
\hline receita & Valor corrente unitário da receita do PRODUTO na BASE no ANO & $\begin{array}{c}\text { M US\$/m3 ou M } \\
\text { US\$/t }\end{array}$ \\
\hline rend ${ }_{\text {uni,cam }}^{\text {pet,pd }}$ & $\begin{array}{l}\text { Fração do rendimento do ÓLEO para o PRODUTO na CAMPANHA na } \\
\text { UNIDADE }\end{array}$ & Fração \\
\hline rend $2_{\text {uni,cam }}^{\text {prd,prt }}$ & $\begin{array}{l}\text { Fração do rendimento do PRODUTO carga para o PRODUTO na } \\
\text { CAMPANHA produzido na UNIDADE }\end{array}$ & Fração \\
\hline
\end{tabular}

Tabela 3.2 - Parâmetros e suas descrições

As variáveis são todas não-negativas, contínuas e estão descritas na tabela abaixo. Seus domínios, com exceção das variáveis de investimento, pertencem ao intervalo $[0,+\infty]$, sendo em alguns casos mais limitados, conforme as restrições descritas ao longo desta seção. As variáveis de investimento têm seus domínios definidos no intervalo $[0,1]$, também de maneira contínua. 
Defini-las como variáveis binárias é uma possibilidade condizente com a realidade, porém nesta dissertação, basicamente por questões de praticidade, optou-se por trabalhar com um modelo puramente linear e contínuo.

\begin{tabular}{|c|c|}
\hline \multicolumn{2}{|r|}{ Variáveis } \\
\hline Abreviatura & Descrição \\
\hline$D E G_{\text {ref,ano }}^{p r d, p r t}$ & Volume do PRODUTO degradado para o PRODUTO' na REFINARIA no ANO \\
\hline$D G M_{\text {fon,ano }}^{\text {pet,ptl }}$ & Volume do PETRÓLEO utilizado para formar o PETRÓLEO' (mistura) na FONTE no ANO. \\
\hline$D M M_{\text {cam,ano }}^{\text {pet,uni }}$ & Volume do PETRÓLEO (mistura) separado na UNIDADE na CAMPANHA no ANO \\
\hline$D M O_{\text {cam,ano }}^{\text {pet,uni }}$ & Volume do PETRÓLEO processado na UNIDADE na CAMPANHA no ANO \\
\hline$D M P_{\text {cam,ano }}^{\text {prd,uni }}$ & Volume do PRODUTO processado na UNIDADE na CAMPANHA no ANO \\
\hline$F X O_{\text {sen,ano }}^{\text {pet,arc }}$ & Volume do PETRÓLEO no ARCO no SENTIDO no ANO \\
\hline$F X P_{\text {sen,ano }}^{\text {prd,arc }}$ & Volume do PRODUTO no ARCO no SENTIDO no ANO \\
\hline$I U C_{\text {uni,ano }}$ & Indica investimento na capacidade de processamento da UNIDADE no ANO \\
\hline$I V A_{\text {arc,ano }}$ & Indica investimento no ARCO no ANO \\
\hline$I V N_{\text {prj,ano }}$ & Indica investimento no PROJETO no ANO \\
\hline$M E P_{b a s, \text { ano }}^{\text {prd }}$ & Volume do PRODUTO atendido pela Petrobrás na BASE no ANO \\
\hline$M O_{\text {por,ano }}^{j, p e t}$ & Volume do PETRÓLEO adquirido do PORTO na faixa (j) no ANO \\
\hline$M O V_{\text {fai, ano }}^{\text {ter }}$ & $\begin{array}{l}\text { Volume total de petróleo e derivados movimentado no TERMINAL na } \\
\text { FAIXASOBRESTADIA no ANO }\end{array}$ \\
\hline$M P_{\text {frt,ano }}^{j, p r d}$ & Volume do PRODUTO importado da FRENTE na faixa (j) no ANO \\
\hline$X O_{\text {frt,ano }}^{j, p e t}$ & Volume do PETRÓLEO exportado para a FRENTE na faixa (j) no ANO \\
\hline$X P_{\text {frt,ano }}^{j, p r d}$ & Volume do PRODUTO exportado para a FRENTE na faixa (j) no ANO \\
\hline
\end{tabular}

Tabela 3.3 - Variáveis e suas descrições 
A Função Objetivo deseja maximizar o Resultado, que está dividido entre Receita e Custo, ano a ano, trazidos a valor presente:

Max Resultado $=\sum_{\text {ano }}$ fatdesc $_{\text {ano }} \times\left[\right.$ ReceitaOp $_{\text {ano }}-$ CustoOp $\left._{\text {ano }}\right]$

Há três formas de geração da Receita, como pode ser visto em (3.2): atendimento da demanda de produto nas bases, onde cada produto possui uma receita associada; exportação de petróleo para as frentes, onde cada petróleo possui um preço de exportação associado; e exportação de produto para as frentes, onde cada produto possui um preço de exportação associado.

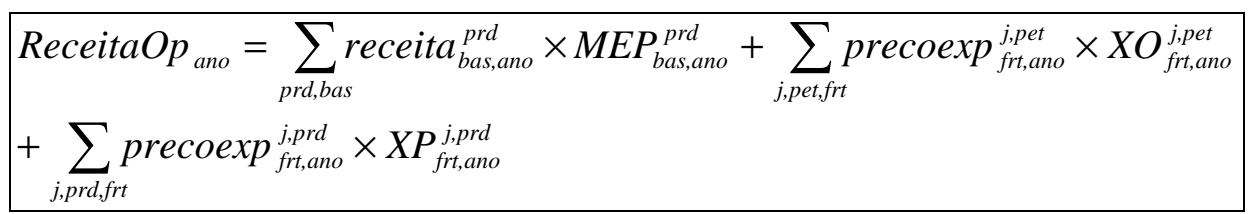

Já o Custo resulta das seguintes operações, na ordem em que aparecem em (3.3): processamento de produto nas unidades, considerando um custo unitário de operação; fluxo de petróleo nos arcos, considerando um custo unitário de transporte; fluxo de produto nos arcos, considerando um custo unitário de transporte; investimento na capacidade de carga das unidades; custo fixo de ampliação de carga das unidades em anos anteriores; investimento na implantação / ampliação de arcos; investimento e custo de operação de projetos; movimentação de petróleo e derivados nos terminais, associada a um custo de sobre-estadia; importação de petróleo nos portos, associada a um preço de importação; e importação de produto nas frentes, associada a um preço de importação.

$$
\begin{aligned}
& \text { CustoOP } p_{\text {ano }}=\sum_{\text {prd,uni,cam }} \text { custoop } 3_{\text {prd,uni }} \times D M P_{\text {cam, ano }}^{\text {prd, }}+\sum_{\text {pet,arc,sen }} \text { custoop } 2_{\text {arc,sen,ano }} \times F X O_{\text {sen,ano }}^{\text {pet,arc }} \\
& +\sum_{\text {prd,arc,sen }} \text { custoop } 2_{\text {arc,sen, ano }} \times F X P_{\text {sen,ano }}^{\text {prd,arc }}+\sum_{\text {uni }} \text { custoinvcarga } a_{\text {uni,ano }} \times I U C_{\text {uni,ano }} \\
& +\sum_{\text {uni,ana|ana } \leq \text { ano }} \text { custoamp }_{\text {uni }} \times I U C_{\text {uni,ana }}+\sum_{\text {arc }} \text { custoinv }_{\text {arc,ano }} \times I V A_{\text {arc,ano }}
\end{aligned}
$$

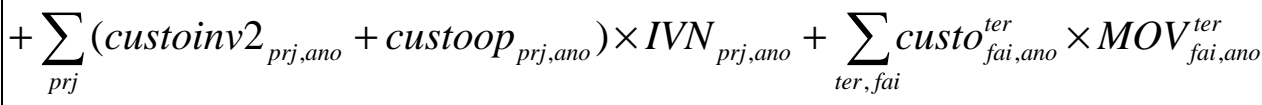

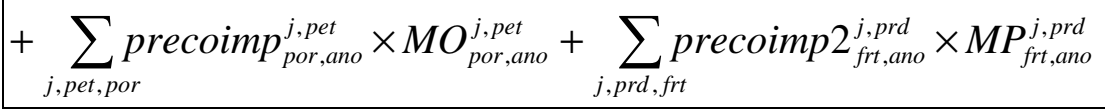

Além disso, o modelo conta com as seguintes restrições: 
- Balanço do petróleo na fonte no ano: Além de produzir determinados tipos de petróleo, algumas fontes podem escoar misturas dos óleos produzidos (na mesma proporção em que são produzidos). Neste caso, o balanço deve ser feito para cada petróleo "componente", e também para o petróleo "mistura". Tem-se portanto que, a quantidade de um petróleo que sai da fonte via fluxo nos arcos, mais a quantidade daquele petróleo escoado em mistura (caso tal petróleo seja um componente), deve ser igual à quantidade produzida daquele petróleo, mais o que entra na fonte via fluxo nos arcos, mais a quantidade de mistura escoada (caso tal petróleo seja uma mistura).

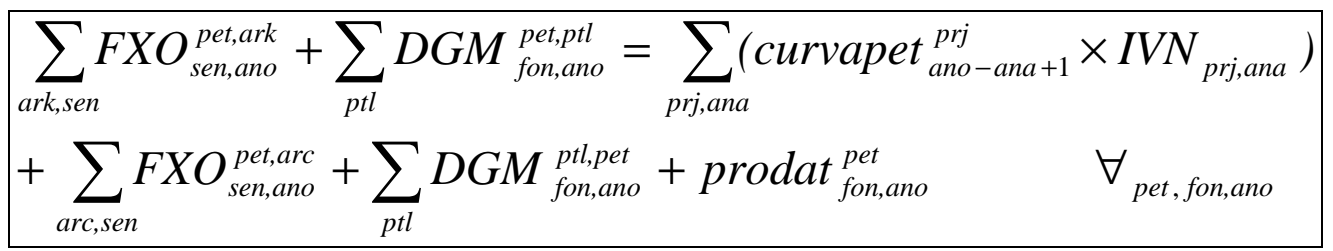

Onde (FON) é destino de (ARC) e origem de (ARK), e (ANA) $\leq$ (ANO)

- Balanço do petróleo no porto no ano: O petróleo que entra no porto via importação, sai do porto via fluxo nos arcos.

$\sum_{j} M O_{\text {por,ano }}^{j, \text { pet }}=\sum_{\text {arc,sen }} F X O_{\text {sen,ano }}^{\text {pet,arc }} \quad \forall_{\text {pet,por,ano }}$

Onde (POR) é origem de (ARC)

- Balanço do petróleo no terminal no ano: A quantidade de petróleo que entra no terminal via fluxo nos arcos é equivalente a quantidade que sai, também via fluxo nos arcos.

$\sum_{\text {arc,sen }} F X O_{\text {sen,ano }}^{\text {pet,arc }}=\sum_{\text {ark,sen }} F X O_{\text {sen,ano }}^{\text {pet,ark }} \quad \forall_{\text {pet,ter,ano }}$

Onde(TER) é destino de (ARC) e origem de (ARK)

- Balanço do petróleo na refinaria no ano: Na refinaria, o petróleo é processado nas unidades de processamento, em diferentes campanhas. Campanhas representam as possíveis modalidades operacionais da unidade. Em cada campanha, o objetivo da operação é diferente, e com isto os rendimentos nos diversos produtos também se alteram. Além do processamento, o petróleo também pode ser "separado" (em seus 
componentes) nas unidades, caso represente uma mistura de óleos. Tem-se então que, a quantidade de petróleo que entra na refinaria via fluxo nos arcos, mais a quantidade de misturas separadas em petróleo (componente), é equivalente à quantidade que sai via fluxo nos arcos, mais a quantidade de petróleo processada nas unidades, mais a quantidade de petróleo (mistura) separada em componentes.

$$
\begin{array}{|lc|}
\sum_{\text {arc,sen }} F X O_{\text {sen,ano }}^{\text {pet,arc }}+\sum_{\text {ptl,uni,cam }}\left(f a t 2_{p t l, \text { ano }}^{\text {pet }} \times D M M_{\text {cam,ano }}^{\text {ptl,uni }}\right)=\sum_{\text {ark,sen }} F X O_{\text {sen,ano }}^{\text {pet,ark }} \\
+\sum_{\text {uni,cam }} D M O_{\text {cam,ano }}^{\text {pet,uni }}+\sum_{\text {uni,cam }} D M M_{\text {cam,ano }}^{\text {pet,uni }} & \forall_{\text {pet,ref,ano }} \\
\hline
\end{array}
$$

Onde (REF) é destino de (ARC) e origem de (ARK) e (UNI) pertence a (REF)

- Totalização da demanda do petróleo na frente no ano: O petróleo que entra na frente via fluxo nos arcos, sai via exportação.

$$
\sum_{\text {arc,sen }} F X O_{\text {sen,ano }}^{\text {pet,arc }}=\sum_{j} X O_{\text {frt,ano }}^{j, \text { pet }} \quad \forall_{\text {pet,frt,ano }}
$$

Onde (FRT) é destino de (ARC)

- Balanço do produto na fonte no ano: A quantidade de produto que sai da fonte via fluxo nos arcos é menor ou igual ao que é produzido na fonte, mais o que entra via fluxo nos arcos.

$$
\begin{array}{|ll}
\sum_{\text {ark,sen }} F X P_{\text {sen,ano }}^{\text {prd,ark }} \leq \sum_{\text {prj,ana }}\left(\text { curvaprod }_{\text {ano-ana }+1}^{\text {prd,prj }} \times I V N_{\text {prj,ana }}\right) \\
+ \text { prodat } 2_{\text {fon, ano }}^{\text {prd }}+\sum_{\text {arc,sen }} F X P_{\text {sen,ano }}^{\text {prd,arc }} & \forall_{\text {prd,fon,ano }}
\end{array}
$$

Onde (FON) é destino de (ARC) e origem de (ARK)

- Balanço do produto no terminal no ano: A quantidade de produto que entra via fluxo nos arcos é equivalente a quantidade que sai, também via fluxo nos arcos.

$$
\sum_{\text {arc, sen }} F X P_{\text {sen,ano }}^{\text {prd,arc }}=\sum_{\text {ark,sen }} F X P_{\text {sen,ano }}^{\text {prd,ark }} \quad \forall_{\text {pet,ter,ano }}
$$

Onde (TER) é destino de (ARC) e origem de (ARK) 
- Balanço do produto na refinaria no ano: Em uma refinaria, parte da produção das unidades é consumida internamente, como carga para outras unidades de processo ou mesmo para geração de energia (consumo próprio). Assim, há produção das unidades que não virá a ser produção da refinaria. Também podem ocorrer degradações misturas de correntes com o objetivo de especificar produtos entregues ao mercado. Tem-se portanto que, a quantidade de produto que entra na refinaria via fluxo nos arcos, mais a quantidade de produto produzida via degradação de outros produtos, mais a quantidade de produto produzido no processamento de petróleo ou de outros produtos nas unidades, é equivalente à quantidade de produto processada nas unidades, mais a quantidade de produto que sai via fluxo nos arcos, mais a quantidade de produto degradada em outros.

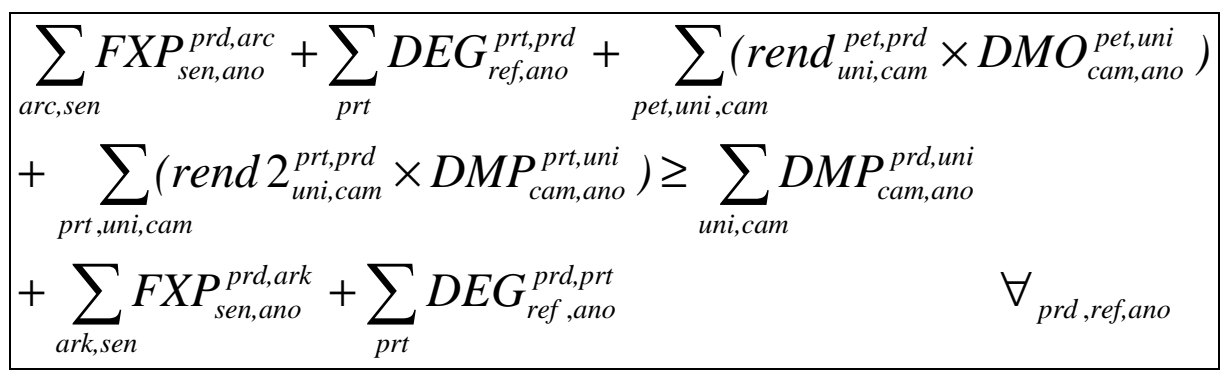

Onde (REF) é destino de (ARC) e origem de (ARK) e (UNI) pertence a (REF)

- Balanço do produto na base no ano: A quantidade de produto que entra na base via fluxo nos arcos é igual ao que sai via fluxo nos arcos, mais o que é utilizado para atender à demanda de produto na base.

$$
\sum_{\text {arc, sen }} F X P_{\text {sen,ano }}^{\text {prd,arc }}=\sum_{\text {ark,sen }} F X P_{\text {sen,ano }}^{\text {prd,ark }}+M E P_{\text {bas,ano }}^{\text {prd }} \quad \forall_{\text {prd,bas,ano }}
$$

Onde (BAS) é destino de (ARC) e origem de (ARK)

- Totalização da importação do produto na frente no ano: O produto que entra na frente via importação, sai via fluxo nos arcos.

$$
\sum_{\text {arc, sen }} F X P_{\text {sen,ano }}^{\text {prd,arc }}=\sum_{j} M P_{\text {frt,ano }}^{j, \text { prd }} \quad \forall_{\text {prd,frt,ano }}
$$

Onde (FRT) é origem de (ARC)

- Totalização da demanda do produto na frente no ano: $\mathrm{O}$ produto que entra na frente via fluxo nos arcos, sai via exportação. 
$\sum_{\text {arc,sen }} F X P_{\text {sen,ano }}^{\text {prd,arc }}=\sum_{j} X P_{\text {frt,ano }}^{j, p r d} \quad \forall_{\text {prd,frt,ano }}$

Onde (FRT) é destino de (ARC)

- Limite de fluxo no arco no ano: Ao longo das equações, pode ser observado que as variáveis de fluxo nos arcos também são indexadas por sentido. Isto quer dizer, na prática, que os arcos podem ser operados tanto no sentido normal como no sentido inverso, mas com capacidades diferentes. Esta diferença é expressa através de um fator multiplicativo, conforme abaixo. Portanto, o fluxo total de petróleo e produtos no arco está limitado à capacidade do arco, considerando possibilidade de ampliação ou desinstalação.

$$
\begin{array}{|l}
\sum_{\text {pet,sen }}\left(\frac{\text { fat }_{\text {pet,arc }}}{\text { fatinv }_{\text {arc,sen }}} \times F X O_{\text {sen,ano }}^{\text {pet,arc }}\right)+\sum_{\text {prd,sen }} \frac{F X P_{\text {sen,ano }}^{\text {prd,arc }}}{\text { fatinv } v_{\text {arc }, \text { sen }}} \leq \text { capat }_{\text {arc }} \\
+ \text { capad }_{\text {arc }} \times \sum_{\text {ana }} I V A_{\text {arc,ana }}-\text { capat }_{\text {arc }} \times \sum_{\text {ark,ana }} I V A_{\text {ark,ana }} \quad \forall_{\text {arc,ano }}
\end{array}
$$

Onde (ANA) é anterior a (ANO) e (ARC) é desinstalado quando (ARK) é implantado

- Limite máximo de capacidade da unidade no ano: A quantidade de carga total processada na unidade deve ser menor ou igual à capacidade máxima da unidade, considerando a possibilidade de desinstalação da mesma.

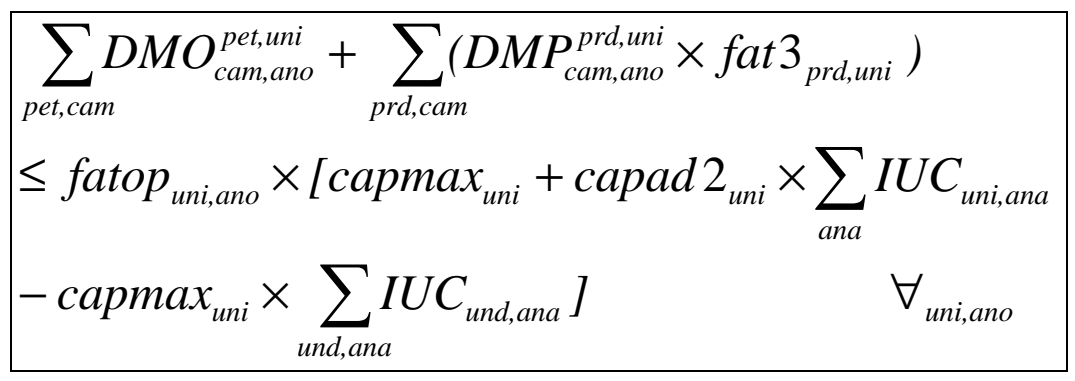

Onde (ANA) é anterior ou igual a (ANO) e (UNI) é desinstalada quando (UND) é instalada

- Limite mínimo de capacidade na unidade no ano: A quantidade de carga total processada na unidade deve ser maior ou igual à capacidade mínima da unidade, considerando a possibilidade de desinstalação da mesma. 


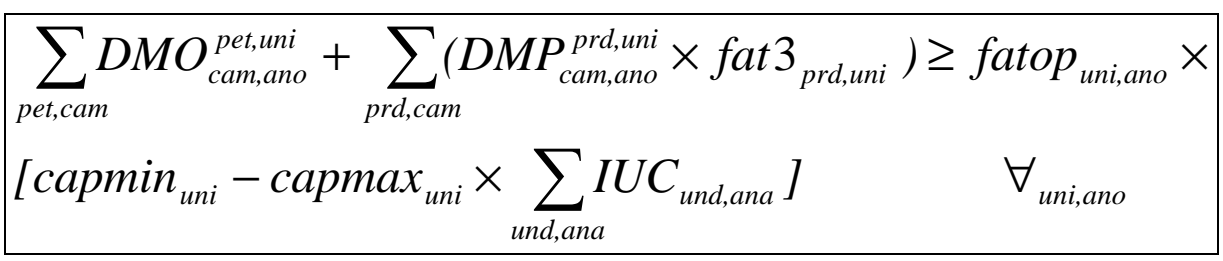

Onde (ANA) é anterior ou igual a (ANO) e (UNI) é desinstalada quando (UND) é instalada

- Limite mínimo de carga do petróleo na refinaria no ano: A quantidade de petróleo processado nas unidades e campanhas da refinaria, deve ser maior ou igual ao volume mínimo requerido na refinaria.

$$
\sum_{\text {uni,cam }} D M O_{\text {cam,ano }}^{\text {pet,uni }} \geq \min _{\text {ref,ano }}^{\text {pet }} \quad \forall_{\text {pet,ref ,ano }}
$$

Onde (UNI) pertence a (REF)

As restrições a seguir impedem que seja implantado mais do que $100 \%$ de cada investimento ao longo dos anos:

$$
\begin{array}{ll}
\sum_{\text {ano }} I V N_{\text {prj,ano }} \leq 1 \quad \forall_{\text {prj }} & \sum_{\text {ano }} I V A_{\text {arc,ano }} \leq 1 \quad \forall_{\text {arc }} \\
\sum_{\text {ano }} I U C_{\text {uni,ano }} \leq 1 \quad \forall_{\text {uni }} &
\end{array}
$$

As restrições a seguir forçam os investimentos vinculados entre si:

$$
I U C_{\text {uni,ano }}=I U C_{\text {und,ano }} \quad \forall_{\text {uni,und,ano }}
$$

Onde (UNI) é instalada quando (UND) é instalada

$$
I V A_{\text {arc }, \text { ano }}=I V A_{\text {ark,ano }} \quad \forall_{\text {arc,ark, ano }}
$$

Onde (ARC) é instalado quando (ARK) é instalado

- Limites de atendimento do mercado do produto na base no ano: $\mathrm{O}$ atendimento da demanda de produto na base deve respeitar limites mínimo e máximo.

$$
\operatorname{mermin}_{\text {bas,ano }}^{\text {prd }} \leq M E P_{\text {bas,ano }}^{\text {prd }} \leq \text { mermax }_{\text {bas,ano }}^{\text {prd }} \quad \forall_{\text {prd,bas,ano }}
$$

Onde o atendimento da demanda por fontes externas é considerado 
- Totalização da movimentação total no terminal no ano: A movimentação no terminal é a soma do que entra e sai do terminal, de petróleo e produtos, via fluxo nos arcos.

$\sum_{\text {pet,arc, sen }} F X O_{\text {sen,ano }}^{\text {pet,arc }}+\sum_{\text {arc,sen }} F X P_{\text {sen,ano }}^{\text {prd,arc }}=\sum_{\text {fai }} M O V_{\text {fai,ano }}^{\text {ter }} \quad \forall_{\text {ter,ano }}$

Onde (TER) é destino ou origem de (ARC)

Por último, as restrições a seguir definem os bounds das variáveis de importação e exportação (de petróleo e produtos); e movimentação no terminal:

\begin{tabular}{|c|c|}
\hline$M O_{\text {por,ano }}^{j, \text { pet }} \leq$ oferta $2_{\text {por,ano }}^{j, \text { pet }}$ & $\forall_{j, p e t, p o r, \text { ano }}$ \\
\hline$M P_{\text {frt,ano }}^{j, p r d} \leq$ oferta $_{\text {frt,ano }}^{j, p r d}$ & $\forall_{j, p r d}$, \\
\hline
\end{tabular}

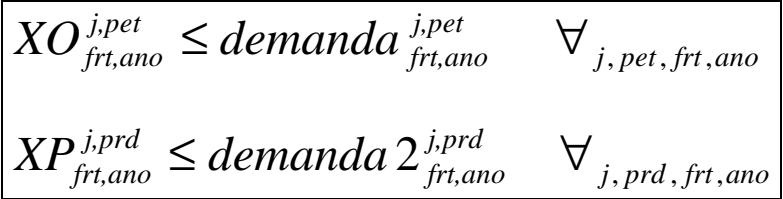

$M O V_{\text {fai,ano }}^{\text {ter }} \leq \max _{\text {fai,ano }}^{\text {ter }} \quad \forall_{\text {ter, fai,ano }}$

\section{2. \\ Versão Estocástica}

Todo o equacionamento mostrado na seção anterior vale também na versão estocástica. Basicamente, a única alteração é a inclusão do conjunto de cenários, que passa a indexar algumas das entidades (parâmetros estocásticos e variáveis de segundo estágio) do modelo.

Não há necessidade, portanto, de repetir todo o equacionamento com as devidas modificações; basta identificar os parâmetros que passam a ser estocásticos e classificar as variáveis em primeiro e segundo estágio, para indexá-los também por cenário. Naturalmente, esta indexação é propagada para as restrições que englobam tais entidades.

O conjunto de cenários, então, é representado pelo índice cen . Os parâmetros que recebem este índice estão sob a ação das incertezas mais significativas do modelo. 
No caso: incertezas relacionadas aos preços; incertezas em relação à demanda de mercado; e incertezas relacionadas à produção de petróleo e produtos nas fontes. A tabela abaixo identifica os parâmetros estocásticos, já com a inclusão do índice de cenários.

\begin{tabular}{|c|c|c|}
\hline \multicolumn{3}{|c|}{ Parâmetros Estocásticos } \\
\hline Preços & Demanda & Produção \\
\hline $\operatorname{precoexp}_{j, p e t, \text { frt,ano }}^{\text {cen }}$ & mermax $_{\text {prd bas,ano }}^{c e n}$ & prodat $_{\text {pet }, \text { fon, ano }}^{\text {cen }}$ \\
\hline precoexp $2_{j, p r d, \text { frt,ano }}^{c e n}$ & $\operatorname{mermin}_{p r d, b a s, a n o}^{c e n}$ & prodat $2_{p r d, f o n, a n o}^{c e n}$ \\
\hline precoimp ${ }_{j, p e t, \text { frt,ano }}^{\text {cen }}$ & & \\
\hline precoimp $2_{j, \text { prd } \text {,por,ano }}^{\text {cen }}$ & & \\
\hline
\end{tabular}

Tabela 3.4 - Parâmetros Estocásticos

Outros parâmetros também estão sujeitos a incertezas, sendo tratados ou como determinísticos, por não impactarem significativamente o resultado; ou à parte da modelagem estocástica, por questões gerenciais, como é o caso do cronograma (atraso / antecipação da entrada em operação) de projetos e do valor de investimento.

A classificação das variáveis é relativamente simples. O primeiro estágio engloba as decisões tomadas antes da realização das incertezas, ou seja, não há necessidade de indexar as respectivas variáveis por cenário, já que elas assumem o mesmo valor qualquer que seja o cenário.

Tipicamente, as variáveis de primeiro estágio representam decisões tomadas com certa antecedência, portanto demandam planejamento. Uma vez que não se conhece o cenário exato que acontecerá no futuro, a decisão de primeiro estágio deverá ser a melhor para o conjunto de cenários possíveis.

Por outro lado, o segundo estágio engloba as decisões tomadas após a realização das incertezas, e por isso as variáveis devem ser indexadas pelo conjunto de cenários. As variáveis de segundo estágio representam as decisões operacionais do modelo, também chamadas de ações corretivas.

No PLANINV, as decisões que valem para todos os cenários são as decisões de investimento. Então o primeiro estágio engloba as três variáveis de investimento: $I U C_{\text {uni,ano }}, I V A_{\text {uni,ano }}, I V N_{\text {prjano }}$. O segundo estágio, portanto, engloba todas as outras 
variáveis que aparecem na tabela 3.3, que definem a operação do sistema. Não há necessidade de repeti-las aqui, basta ressaltar que as variáveis de segundo estágio devem ser indexadas por cenário.

Da mesma forma, as restrições em que há participação destas variáveis de segundo estágio, devem ser indexadas por cenário. No caso, todas as restrições, exceto a 3.19, a 3.20 e a 3.21, passam a ser indexadas por cenário. Não há necessidade de reapresentá-las com esta pequena modificação. A título de exemplo, a restrição de balanço de produto na fonte (3.9) fica assim:

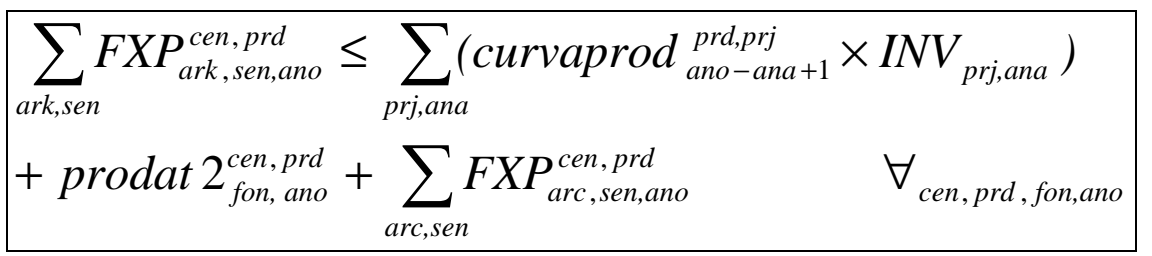

Por fim, a função objetivo, que na versão determinística maximiza o resultado, passa a maximizar o valor esperado do resultado. Na prática, temos o seguinte:

Max Resultado $=\sum_{c e n}\left[\mathrm{P}_{c e n} \times \sum_{\text {ano }}\right.$ fatdesc $_{\text {ano }} \times\left(\right.$ ReceitaOp $_{\text {cen, ano }}-$ CustoOp $\left.\left._{\text {cen, ano }}\right)\right]$

onde $\mathrm{P}_{c e n}$ é a probabilidade de ocorrência do cenário.

No capítulo 4, fala-se um pouco mais sobre a construção do Cenário Base e geração dos cenários estocásticos.

\section{3.}

\section{Decomposição Multicortes}

Feita a classificação das variáveis entre primeiro e segundo estágio, é possível montar problemas menores que podem ser resolvidos separadamente, seguindo a lógica descrita com mais detalhes matemáticos no capítulo 2. Em suma, o modelo decomposto é resolvido de forma iterativa, onde a solução para o primeiro estágio é fixada e utilizada para solucionar o segundo estágio, para cada cenário. Cortes de otimalidade são gerados e adicionados (na forma de restrições) ao problema de primeiro estágio. 
O problema de primeiro estágio é também chamado de "mestre", e engloba basicamente as restrições e variáveis de investimento, ou seja, que compõem o primeiro estágio.

O processo então se repete, chegando-se a uma nova solução de primeiro estágio, que é fixada e utilizada para solucionar o segundo estágio de cada cenário. Cada cenário é abordado separadamente, o que contorna eventuais limitações de porte do modelo, em problemas chamados de "escravos".

Tem-se portanto, um problema de segundo estágio para cada cenário, que engloba apenas as restrições e variáveis de operação, ou seja, que compõem o segundo estágio. As variáveis de primeiro estágio também aparecem, mas fixadas com o valor obtido na última solução do problema de primeiro estágio.

No caso de haver inviabilidade no segundo estágio de algum dos cenários, é necessário medir tal inviabilidade com o auxílio de variáveis de folga. Gera-se então, um corte de viabilidade, que é adicionado (na forma de restrição) ao problema de primeiro estágio, e a iteração é reiniciada.

O processo iterativo continua até um determinado critério de convergência ser atingido. Isto ocorre quando os bounds (upper e lower) chegam a uma distancia suficientemente próxima, um do outro. O Upperbound é simplesmente o valor da FO ao final da iteração, calculado pela soma dos investimentos (custos envolvidos nas decisões de primeiro estágio) com o valor médio real da operação nos cenários (receitas e custos provenientes das decisões de segundo estágio). Já o Lowerbound vem da soma dos investimentos com o valor aproximado da operação.

O código abaixo, comentado e com algumas simplificações, ilustra melhor o processo iterativo descrito:

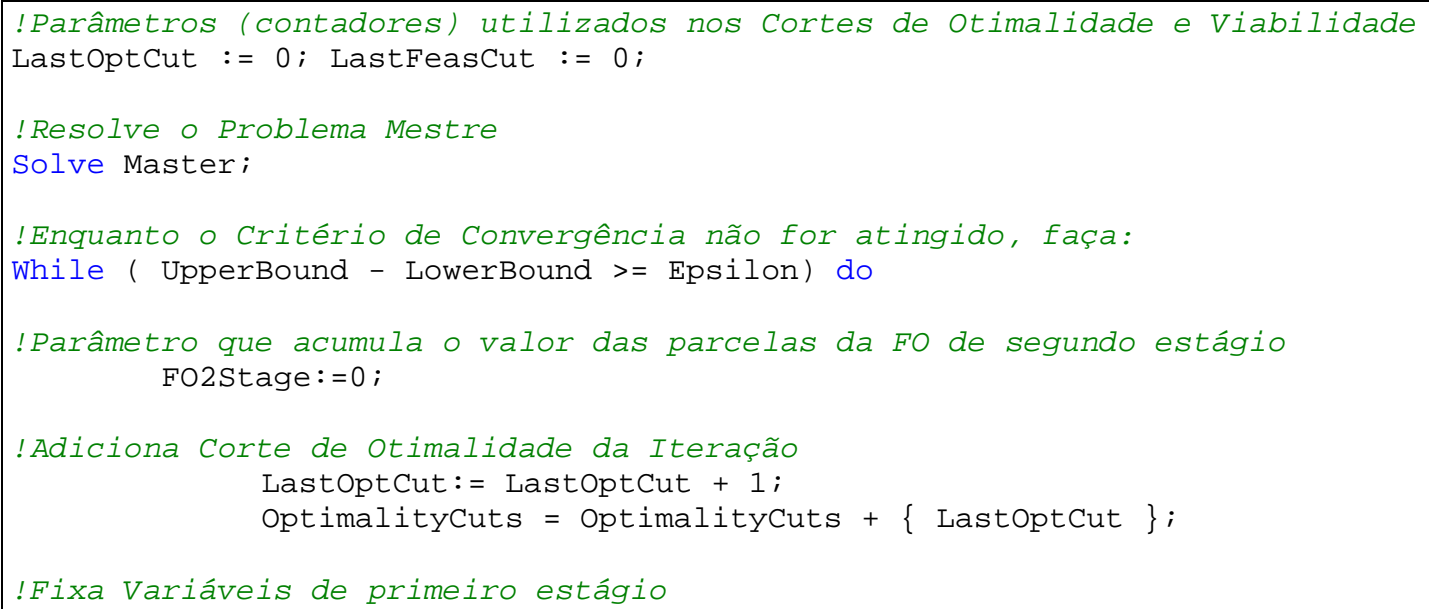




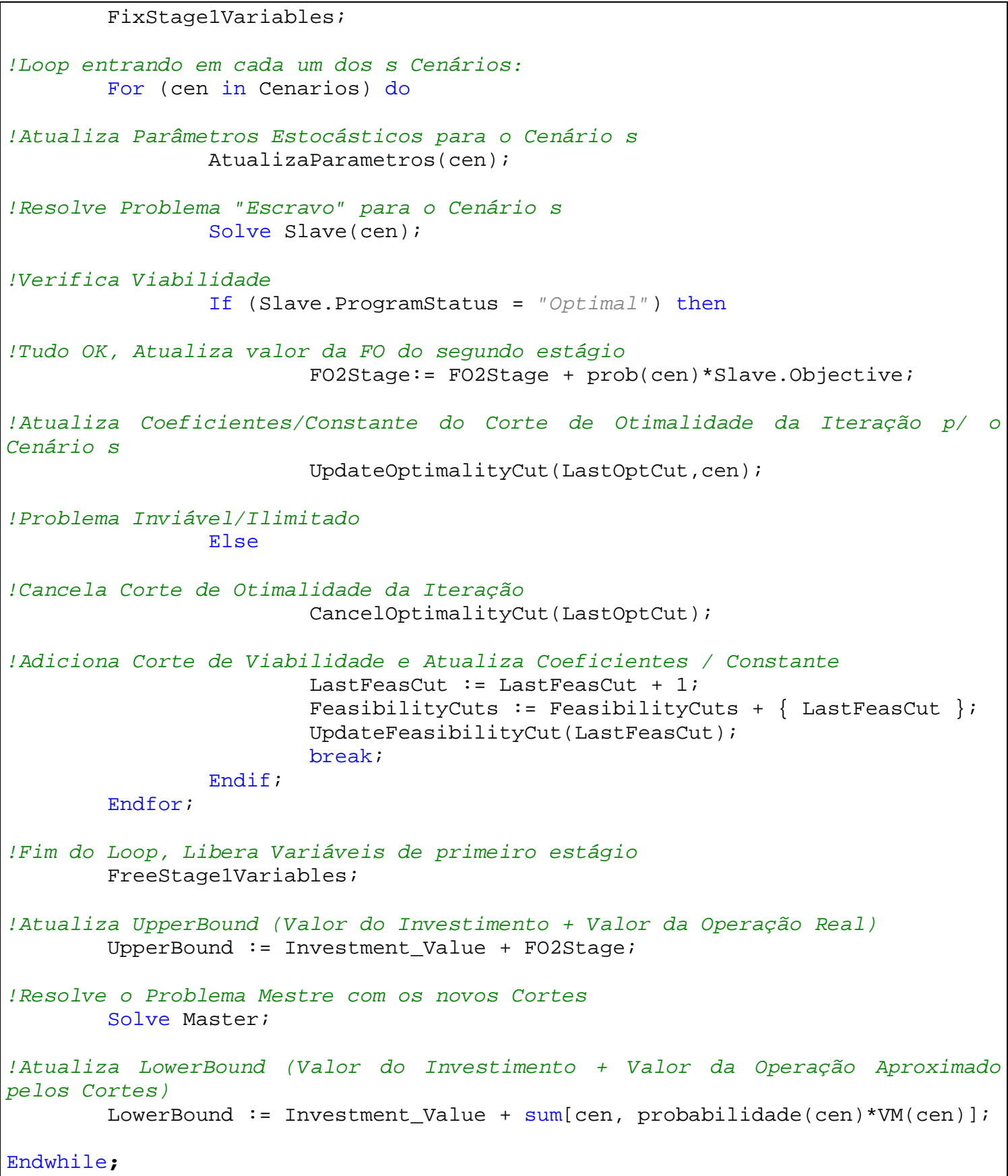




\section{4}

\section{Testes e Resultados}

Esta seção detalha todo o procedimento dos testes realizados, mostrando os resultados considerados mais relevantes. Basicamente, a seção é dividida em duas partes: Cenário Base e Cenários Estocásticos. A primeira trata do caso determinístico, dando uma idéia de porte, e principais informações consideradas pelo modelo. Já a segunda trata do caso estocástico, e expõe os resultados de dois grupos de testes: as rodadas puramente estocásticas, e as rodadas decompostas. Antes disso, porém, a configuração do ambiente de testes é brevemente detalhada.

\section{1.}

\section{Configuração do Ambiente Utilizado}

O modelo e suas evoluções foram implementados no software AIMMS (Advanced Integrated Multidimensional Modeling Software - Bisschop e Roelofs, 2007), e os testes / resultados computacionais descritos neste capítulo foram realizados em um servidor com 2 processadores Intel ${ }^{\circledR}$ Xeon ${ }^{\circledR}$ - X5650 2.67GHz e 94Gb RAM total. Para fins comparativos, alguns dos testes também foram rodados em um desktop com processador Intel ${ }^{\circledR}$ Core TM 2 Duo - E7200 2.53GHz e 4Gb RAM.

O solver utilizado para a otimização do modelo foi o Gurobi 5.0, com método de resolução baseado no algoritmo Barreira. O solver Cplex 12 também foi testado, mas não foi constatada nenhuma diferença de performance significativa.

A principal diferença no tempo de otimização ocorreu quando o método de resolução foi alterado para o Dual Simplex. Houve grande perda de eficiência, e o tempo de solução chegou a mais do que dobrar em alguns casos.

O critério de convergência, utilizado para determinar o fim do processo iterativo de solução do modelo decomposto, foi especificado com base no VSS médio. Concluiuse que $10 \%$ do VSS médio (em torno de $1 \mathrm{MM}$ ) é um valor aceitável para indicar a convergência do modelo. 


\section{2.}

\section{Cenário Base}

Uma razoável quantidade de tempo foi dedicada à realização de ajustes e testes prévios, com o intuito de especificar uma configuração ideal para o Cenário Base. Entende-se por configuração ideal, a definição de determinados parâmetros, como horizonte de tempo e quantidade / tipo de projetos de investimento possíveis, por exemplo, de modo a deixar o estudo mais coerente com a realidade do que é feito na prática, no dia-a-dia da empresa.

Desta forma, chegou-se a um cenário definido com um horizonte de estudo de 8 (oito) anos e um total de 23 projetos de investimento "livres" (sendo decisão do modelo implantá-los ou não, e quando). Estes projetos podem ser desde instalações / ampliações de unidades em refinarias, até instalações / ampliações de arcos entre nós.

Além disso, o Sistema Petrobras foi representado, no Cenário Base, por aproximadamente: 25 grupos de petróleo (fora todas as possibilidades de mistura); 185 produtos principais (além dos desagregados); 27 fontes de produção; 22 refinarias contendo quase 200 unidades (divididas em 34 tipos diferentes); 54 bases de distribuição; 20 frentes e 10 portos de importação de petróleo; 44 terminais; e ainda 78 possibilidades de transporte, divididas em 7 modais. Para representar as conexões existentes entre os nós, abrindo a possibilidade de movimentação dos mais variados produtos nos diferentes modais, foram necessários cerca de 2500 arcos.

Para cada um dos parâmetros considerados estocásticos, utilizou-se a média dos valores daquele parâmetro nos diferentes cenários disponíveis. Neste sentido, o Cenário Base é uma espécie de cenário médio em relação aos cenários corporativos disponíveis.

Sua otimização determinística gerou um modelo com 211.668 restrições e 637.183 variáveis, e chegou à solução ótima (FO: 128.770,99 MM) em 50 segundos no desktop. Já rodando no servidor, este tempo foi reduzido para 35 segundos. 


\section{3.}

\section{Cenários Estocásticos}

\subsection{1.}

\section{Seleção de Cenários}

As rodadas estocásticas foram pré-definidas para 3, 10, 30 e 80 cenários. Cada um destes cenários possui a mesma probabilidade de ocorrência e foi escolhido aleatoriamente, entre um conjunto maior de cerca de 2.000 cenários corporativos.

A geração destes cenários é feita por uma área específica da Companhia, que utiliza-se de determinadas técnicas e conhecimento que não fazem parte do escopo deste trabalho. O modelo já os recebe prontos, na forma de dados de entrada.

A curva de produção de petróleo e derivados, por exemplo, é simulada de forma independente do cenário de preços de petróleo e produtos. Isto significa admitir que o nível de produção de petróleo independe do nível de preço do petróleo no mercado internacional e que a variação simulada estaria relacionada mais fortemente a incertezas geológicas.

Por outro lado, dados de demanda doméstica, que são tratados estocasticamente através de processos de reversão à média, devem manter a dependência histórica entre produtos e os mercados.

Enfim, realizações dos parâmetros estocásticos apresentados no Capítulo 3 - que representam as incertezas mais significativas consideradas no problema - são combinadas, respeitando-se eventuais correlações entre eles, e daí surgem os cenários corporativos.

A escolha aleatória dos cenários de teste, a partir deste conjunto de cenários corporativos, é que poderia ser tema de discussão. Sendo necessário para isso, o estudo de algumas técnicas de redução de cenários. Uma idéia seria basicamente, através de determinadas métricas, agrupar os cenários corporativos considerados mais "próximos" entre si, elegendo um representante e atribuindo-lhe certa probabilidade de ocorrência. Desta forma, o nível de agrupamento seria maior ou menor, de acordo com a quantidade de cenários representativos que se desejasse utilizar em cada rodada.

Alguns dos trabalhos que podem ser encontrados na literatura abordando técnicas de geração / redução de cenários são: Heitsch e Römisch (2005); Mitra (2006); Di Domenica et al. (2009); Kleywegt et al. (2002); Linderoth e Wright (2003); Verweij et al. (2003); Santoso et al. (2005); Schütz et al. (2009). 


\subsection{2. Rodadas Estocásticas}

A primeira rodada foi definida com 3 cenários. O porte do problema estocástico atingiu as 683.455 restrições e 1.909 .647 variáveis.

Utilizando-se a solução determinística do Cenário Base (para as variáveis de primeiro estágio) para resolver cada um dos 3 cenários, chegou-se ao EEV de 126.405,15 MM. Ou seja, este valor é o que o decisor conseguiria sem a necessidade de um modelo estocástico; apenas resolvendo os 3 cenários com a configuração ótima do cenário médio.

Por outro lado, se conhecesse exatamente a realização futura de cada um dos cenários, como se tivesse uma bola de cristal, chegaria à solução WS de 126.409,96 MM. É importante salientar que WS é a melhor solução na teoria, mas no mundo real não é possível obtê-la, dado que não há como prever todos os cenários futuros com exatidão.

A solução para o problema estocástico (RP) então, deve estar sempre em algum ponto entre EEV e WS, sendo EEV $\leq \mathrm{RP} \leq \mathrm{WS}$. No caso dos 3 cenários utilizados, chegou-se ao RP de 126.409,95 MM, muito próximo à informação perfeita.

Temos um EVPI da ordem de 10 mil, o que comparado aos altos valores das soluções, denota a pequena importância de se ter a informação perfeita, dado que a solução estocástica já é suficientemente boa. Da mesma forma, o VSS é da ordem de 4.8 MM, o que já representa um valor razoável de ganho em relação à solução EEV, justificando esforços para se ter a modelagem estocástica.

O solver Gurobi 5.0, no desktop, levou 293 segundos para otimizar o problema estocástico com 3 cenários utilizando o algoritmo Barreira; este número aumentou sensivelmente, para 754 segundos, utilizando o algoritmo Dual Simplex. Já no servidor, o mesmo solver, utilizando o Barreira, otimizou o problema em 156 segundos, contra 402 segundos do Dual Simplex.

O solver Cplex 12 obteve praticamente os mesmos resultados; portanto as rodadas seguintes foram feitas apenas com o solver Gurobi 5.0, utilizando o algoritmo Barreira.

A segunda rodada foi definida com 10 cenários. O porte do problema estocástico atingiu as 2.277.775 restrições e 6.363.292 variáveis.

Chegou-se ao EEV de 117.256,50 MM. A solução espere-e-veja, ou WS, foi de 117.273,58 MM. Já a solução para o problema estocástico, ou RP, foi de 117.273,58 MM, praticamente equivalente à solução WS. 
Desta forma, tivemos um EVPI virtualmente igual a zero. Por outro lado, o VSS chegou aos 17 MM. Esta variação no VSS pode ter acontecido pela inclusão, na rodada, de alguns cenários específicos que contribuíram para isso, dado que a seleção de cenários foi feita de forma aleatória. Mas a tendência, como será observado mais adiante, é que haja convergência do VSS com o aumento do numero de cenários utilizados.

No desktop, o Gurobi 5.0, utilizando o algoritmo Barreira, otimizou o problema em 1.353 segundos. Já no servidor, o problema foi resolvido em 711 segundos.

A terceira rodada foi definida com 30 cenários. O porte do problema estocástico atingiu 6.832.975 restrições e 19.087.992 variáveis.

Chegou-se ao EEV de 132.202,92 MM; a solução WS foi de 132.212,32 MM; e a solução RP, foi de 132.212,32 MM, praticamente equivalente à solução WS.

Desta forma, tivemos um EVPI virtualmente igual a zero. Por outro lado, o VSS foi de 9,4 MM.

O desktop não dispôs de memória suficiente para otimizar o problema com 30 cenários, dado o porte atingido. Já no servidor, o Gurobi 5.0, utilizando o algoritmo Barreira, otimizou o problema em 3.579 segundos.

A quarta e última rodada foi definida com 80 cenários. O porte do problema estocástico atingiu 18.220.975 restrições e 50.899 .742 variáveis.

Chegou-se ao EEV de 131.645,25 MM; a solução WS foi de 131.655,95 MM; e a solução RP não foi obtida, por memória insuficiente dado o porte atingido pelo modelo. Seguindo a lógica das rodadas anteriores é de se esperar um RP bem próximo ao valor WS.

Desta forma, teríamos um EVPI praticamente zerado, ou bem próximo disso. Por outro lado, o VSS seria algo em torno de 10,7 MM.

Como nem o servidor dispôs de memória suficiente para otimizar o problema com 80 cenários, foi feita uma projeção para o tempo de otimização. Ajustando duas funções, uma polinomial e uma de potência, chegou-se ao gráfico abaixo: 


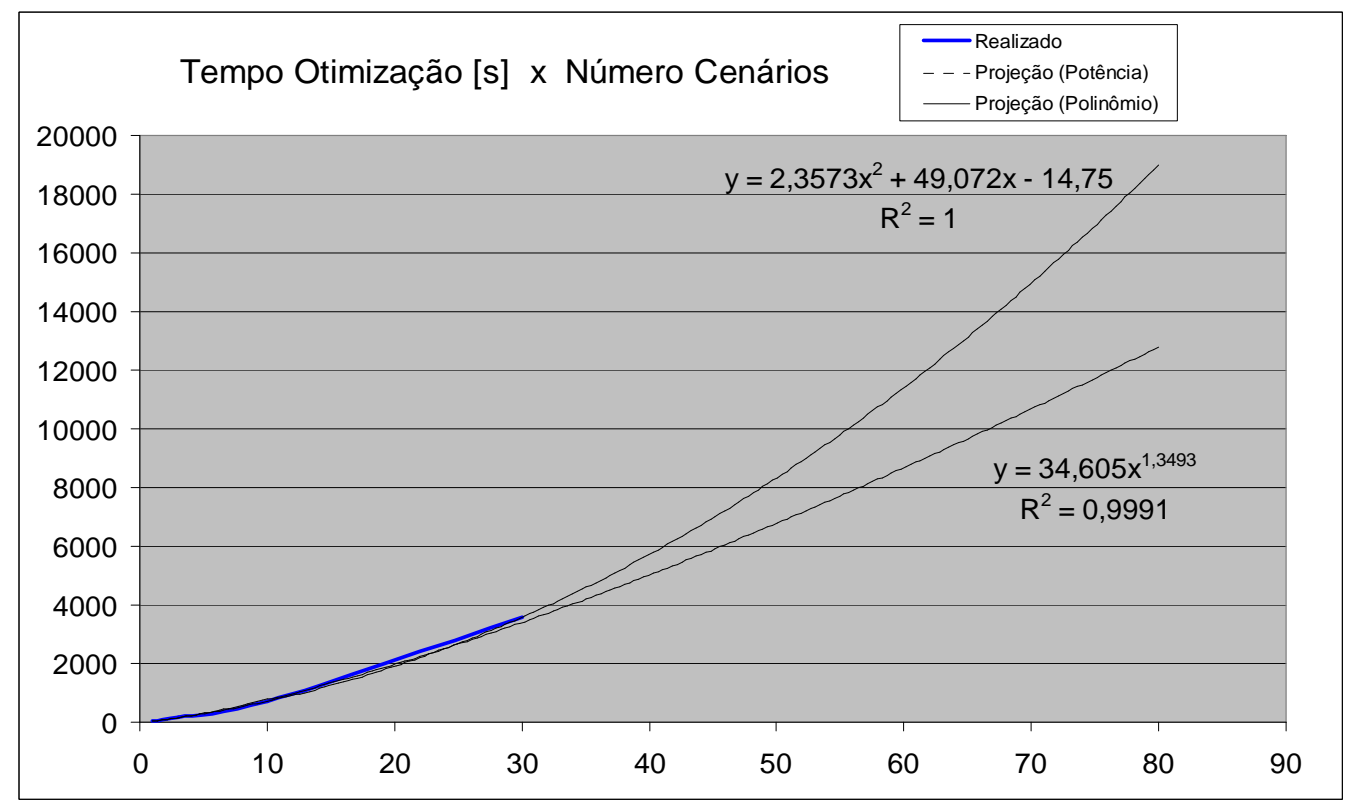

Figura 4.1 - Projeção do tempo de otimização

onde, de acordo com as equações obtidas, estimou-se um tempo de otimização entre 12.793 segundos (projeção por potência) e 18.998 segundos (projeção por polinômio). É importante ressaltar que esses valores são apenas projeções, e as curvas projetadas no gráfico devem ser entendidas meramente como curiosidade.

A tabela abaixo mostra um resumo das quatro rodadas estocásticas feitas:

\begin{tabular}{l|ccccc|}
\multicolumn{1}{c}{} & 1 & 3 & 10 & 30 & 80 \\
\cline { 2 - 6 } \# Restrições & 211.668 & 683.455 & 2.277 .775 & 6.832 .975 & 18.220 .975 \\
\# Variáveis & 637.183 & 1.909 .647 & 6.363 .292 & 19.087 .992 & 50.899 .742 \\
T de otimização (s) & 35 & 156 & 711 & 3579 & 12.793 a 18998 \\
& & & & & \\
Solução RP & & & & & $131.655,95$ \\
Solução EEV & $126.409,95$ & $117.273,58$ & $132.212,32$ & $131.645,25$ \\
VSS & $126.405,15$ & $117.256,5$ & $132.202,92$ & 10,7 \\
\hline & 4,8 & 17,08 & 9,4 & \\
& & & & &
\end{tabular}

Tabela 4.1 - Estatísticas das rodadas estocásticas

onde, os valores em vermelho representam estimativas.

Algumas informações importantes podem ser observadas nesta tabela. Por exemplo, o valor da solução estocástica depende dos cenários utilizados na otimização, 
mas é razoável supor que com o aumento do número de cenários, haja convergência do VSS. Nos casos observados, o valor de convergência seria da ordem de 10 MM.

Observou-se também a necessidade de decompor o modelo, de modo a contornar o problema da insuficiência de memória para a rodada com 80 cenários. Como o porte do modelo estocástico cresce bastante com o número de cenários, o que pode ser verificado de forma mais clara no gráfico da evolução do tempo de otimização, chega um ponto em que investir em um hardware com melhor desempenho não gera ganho justificável. Então uma das alternativas foi implementar as técnicas de decomposição abordadas no capitulo 2 .

\subsection{3. \\ Rodadas Decompostas}

No servidor, o Gurobi 5.0 leva em torno de 1 minuto para resolver o problema de segundo estágio de um cenário qualquer, criar um corte de viabilidade e atualizar os parâmetros estocásticos para o cenário seguinte. Resolvidos todos os cenários, o solver resolve o problema de primeiro estágio já com as novas restrições (cortes), e atualiza os bounds para verificar a convergência, praticamente de forma instantânea.

Ou seja, uma iteração como a descrita no parágrafo acima, leva 1 minuto por cenário utilizado, em média. Então, no caso da primeira rodada, de 3 cenários, em que a convergência foi atingida em 108 iterações; o tempo total de solução foi de aproximadamente 19.440 segundos.

À primeira vista é um tempo indesejavelmente grande, ainda mais se comparado aos 156 segundos da rodada estocástica, para os mesmos 3 cenários. Um incremento de mais de cem vezes.

Mas o fator atenuante é que o ganho da decomposição não está no tempo de solução, e sim na possibilidade de resolver, mesmo que em um tempo bastante grande, problemas que esbarram na limitação da capacidade computacional.

Este foi particularmente o caso da rodada estocástica com 80 cenários. Quando rodado o modelo estocástico completo, ocorre insuficiência de memória; porém quando rodado o modelo decomposto, apesar do tempo despendido, o processo converge e chega-se bem perto da solução ótima, como veremos mais a frente.

Antes disso, seguem alguns resultados das primeiras rodadas decompostas: 


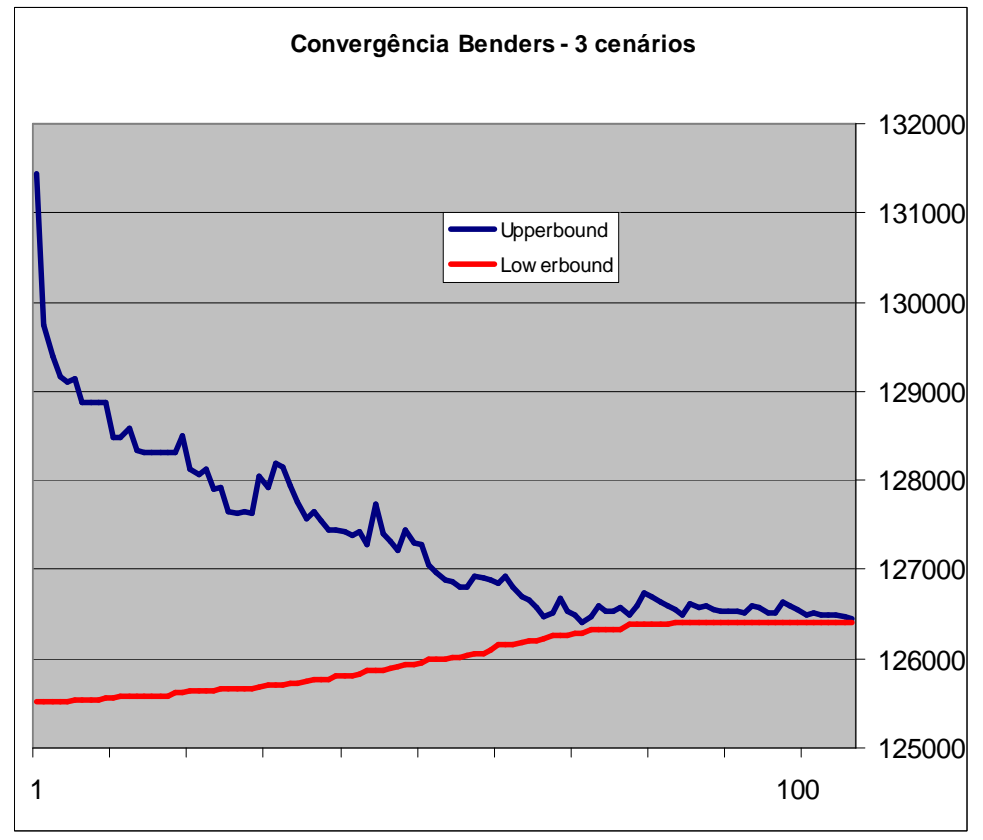

Figura 4.2 - Convergência dos bounds para 3 cenários

A figura acima mostra a convergência dos bounds ao longo do processo iterativo de solução do modelo decomposto na primeira rodada, com 3 cenários. Até atingir o critério de convergência, foram necessárias 108 iterações, realizadas em aproximadamente 19.440 segundos.

A segunda rodada utilizou 10 cenários e atingiu a convergência em 83 iterações, conforme figura abaixo:

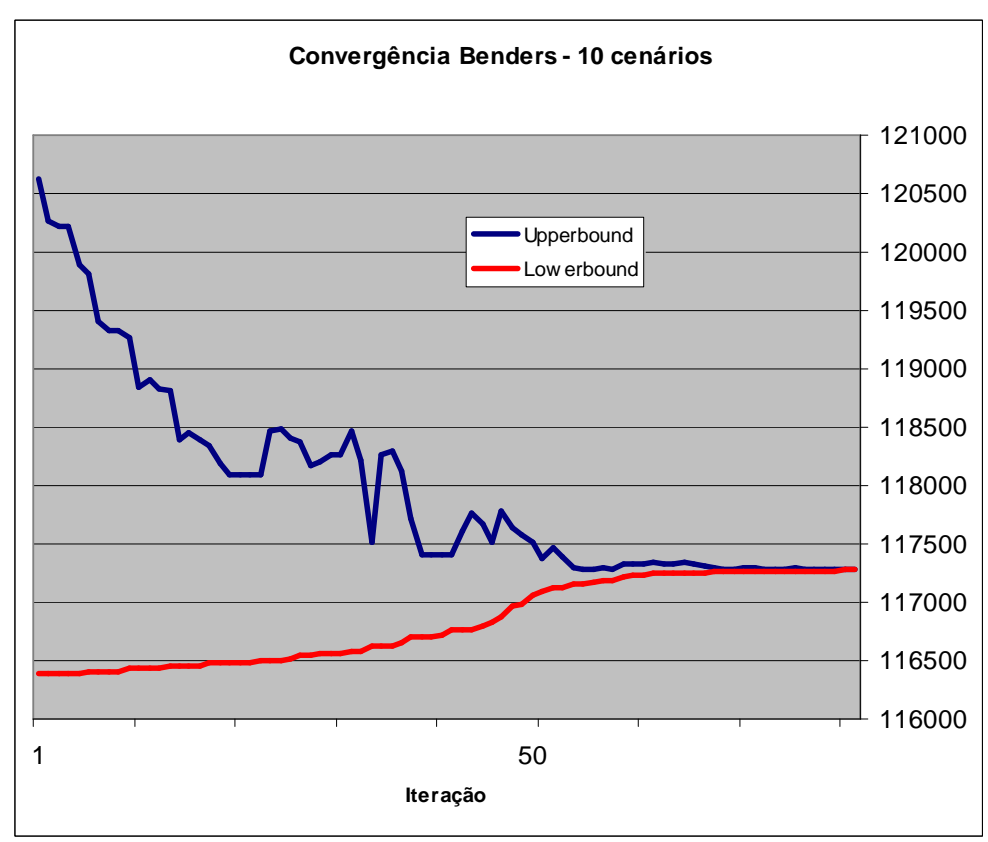




\section{Figura 4.3 - Convergência dos bounds para 10 cenários}

onde é possível observar que, tivesse sido adotado um critério de convergência menos rígido, a resolução da segunda rodada se daria em menos de 70 iterações. Enfim, as 83 iterações resultaram em um tempo total de solução de, aproximadamente, 49.800 segundos.

A terceira rodada foi feita com 30 cenários e atingiu a convergência em 47 iterações, que levou a um tempo total de 84.600 segundos, praticamente 1 dia rodando.

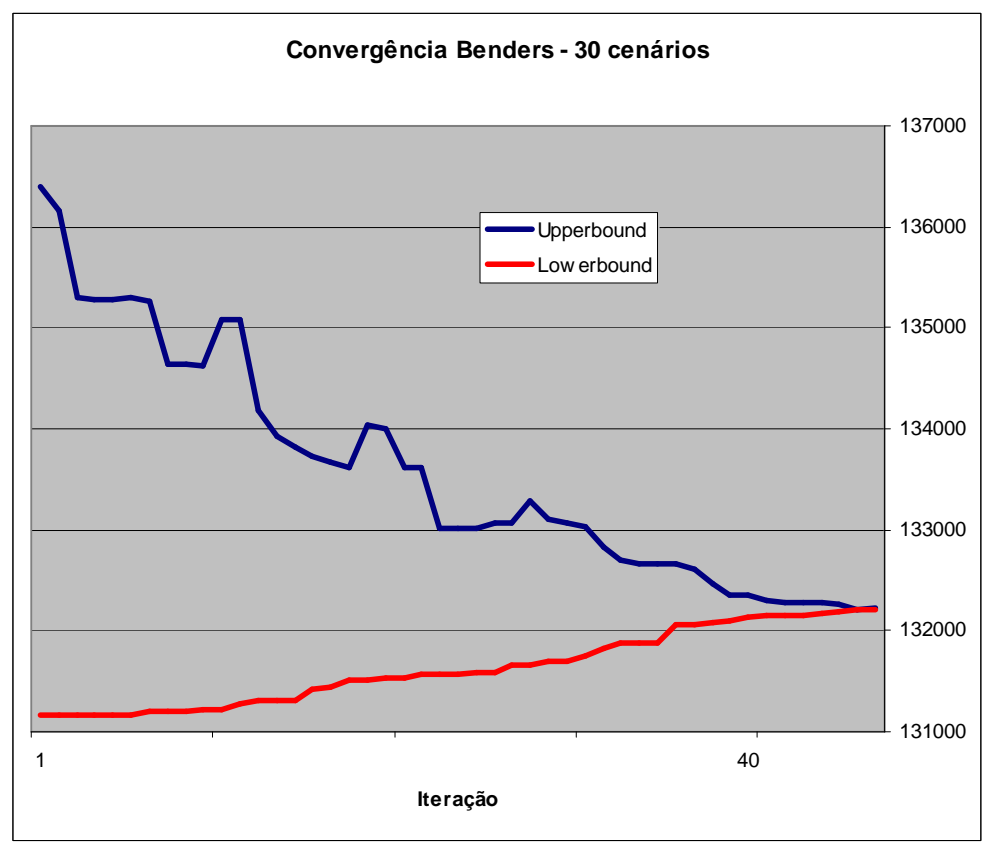

Figura 4.4 - Convergência dos bounds para 30 cenários

Um fato interessante a ser observado é que o número de iterações decai nas rodadas com mais cenários. Talvez pelo método de cortes utilizado (multicortes), quanto maior o número de cenários, mais restrições são adicionadas a cada iteração, o que poderia estar levando à convergência em menos iterações. Apesar disso, o tempo total de solução cresce a cada rodada com mais cenários. Não há garantia de que esse comportamento se repita em outros problemas.

A quarta e última rodada, contemplando 80 cenários, levou 163.200 segundos em 34 iterações para atingir o critério de parada. Importante salientar, novamente, que apesar de uma quantidade razoavelmente grande de tempo para alcançar o critério de convergência estipulado, o método de decompor o modelo é bem sucedido no que se 
propõe. Se antes esbarrava-se nos limites computacionais, agora é possível chegar a uma solução para 80 cenários, bastando para isso haver disponibilidade de tempo.

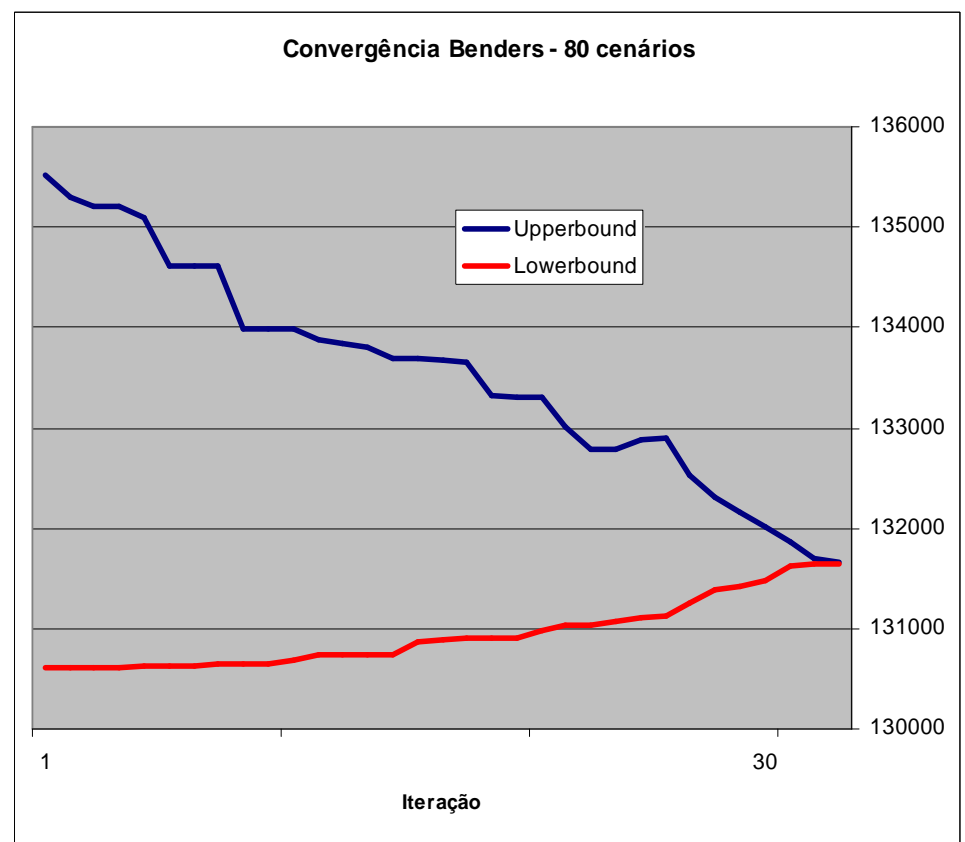

Figura 4.5 - Convergência dos bounds para 80 cenários

Como era de se esperar, os bounds convergiram na direção da solução estocástica estimada na seção anterior, ou seja, em torno de 131.655 MM.

A tabela abaixo mostra um resumo das quatro rodadas decompostas:

\begin{tabular}{l|rrrc|}
\multirow{2}{*}{ \# Iterações } & \multicolumn{1}{c}{3} & 10 & 30 & 80 \\
\cline { 2 - 5 } t de otimização (s) & 108 & 83 & 47 & 34 \\
& & & & \\
& 19.440 & 49.800 & 84.600 & 163.200 \\
\hline
\end{tabular}

Tabela 4.2 - Estatísticas das rodadas estocásticas 


\section{5 \\ Conclusão}

O presente trabalho propôs importantes evoluções em um dos modelos de programação matemática mais antigos, e mais utilizados na Petrobras. O processo decisório de cunho estratégico para a Companhia é fortemente embasado nas indicações e estudos feitos a partir dos resultados que o modelo PLANINV apresenta; então qualquer esforço evolutivo, no sentido aperfeiçoar as técnicas matemáticas utilizadas, pode impactar positivamente tal processo.

Para isso, recorreu-se à literatura, no intuito de se identificar novas tendências e as técnicas que estão em voga nos trabalhos mais atuais, mas sem deixar de lado clássicos da programação matemática, como Beale (1955) ou Dantzig (1955). Primeiramente, foram estudados trabalhos que abordam a cadeia do petróleo, preferencialmente de forma integrada. Em seguida, foi dada ênfase a trabalhos que aplicam técnicas de programação estocástica para lidar com incertezas, não se restringindo à área de petróleo. Por último, o foco foi direcionado ao estudo das técnicas de decomposição, principalmente derivadas do pioneiro trabalho de Benders (1962).

A estrutura do trabalho buscou seguir tal sequiência de estudo. Ou seja, inicialmente foram aplicadas técnicas de programação estocástica em um modelo matemático que busca representar a cadeia do petróleo de forma integrada, de modo a tratar seus parâmetros incertos conjuntamente. A partir daí, começaram a surgir as primeiras limitações em relação ao porte atingido pelo modelo, conforme o número de cenários utilizados para representar as incertezas crescia. Foi então identificada a necessidade de se contornar tais limitações, e as técnicas de decomposição estudadas encontraram justificativa para serem aplicadas e testadas.

Em linha com as expectativas, os resultados mostram que o processo iterativo de resolução do modelo decomposto consegue atingir a convergência com sucesso, nos testes realizados até 80 cenários. O modelo puramente estocástico já havia apresentado dificuldades nos testes com mais de 30 cenários, o que depõe a favor das técnicas de decomposição implementadas. Por outro lado, o processo iterativo demanda um tempo computacional significativamente maior que o modelo estocástico, para resolver 
problemas com o mesmo número de cenários (até 30). Então, passa a ser uma questão de haver tempo suficiente disponível, e/ou do quão imprescindível é incluir um maior número de cenários na otimização.

Além disso, paralelamente a esta questão do numero de cenários $\mathrm{x}$ tempo de otimização, é importante salientar que houve ganho real mensurável em se utilizar o modelo estocástico ante o determinístico, em todos os casos de teste.

Há de se mencionar também, que foi vislumbrada uma boa oportunidade para estudos futuros, inclusive continuação do presente trabalho, na área de redução de cenários. Atualmente, um grande número de cenários corporativos é gerado por uma área específica da Companhia, e dentre estes, alguns cenários foram escolhidos aleatoriamente para os testes. A idéia seria aprimorar este processo de seleção de cenários, de modo que os cenários escolhidos sejam representativos da amostra corporativa. 


\section{Referências Bibliográficas}

Beale, E. (1955). On Minimizing a Convex Function Subject to Linear Inequalities. Journal of the Royal Statistical Society, Series B (Methodological), 17, 2, 173-184.

Benders, J. F. (1962). Partitioning procedures for solving mixed-variables programming problems. Numerische Mathematik, 4: 238-252.

Birge, J.R.; Louveaux, F.V. (1997). Introduction to stochastic programming. New York, NY: Springer.

Bisschop, J.; Roelofs, M. (2007). AIMMS - The User's Guide. Paragon Decision Technology, p. 327.

Carneiro, M.C.T. (2008). Otimização sob incerteza de carteiras de investimentos: aplicação à cadeia integrada de petróleo e derivados. Departamento de Engenharia Industrial, PUC-Rio, Rio de Janeiro. Mestrado.

Carneiro, M.C.T.; Ribas, G.P.; Hamacher, S. (2010). Risk management in the oil supply chain: A CVaR approach. Ind. Eng. Chem. Res., 49:3286-3294.

Cordeau, J.F.; Soumis, F.; Desrosiers, J. (2000). A benders decomposition approach for the locomotive and car assignment problem. Transportation Science, 34(2):133-149.

Dantzig, G. (1955). Linear Programming Under Uncertainty. Management Science, 50, 12 Supplement, 1764-1769.

Di Domenica, N.; Lucas, C.; Mitra, G.; Valente, P. (2009). Scenario generation for stochastic programming and simulation: a modelling perspective. IMA Journal of Management Mathematics, 20:1-38.

Elkamel, A.; Ba-Shammakh, M.; Douglas, P.; Croiset, E. (2008). An Optimization Approach for Integrating Planning and $\mathrm{CO} 2$ Emission Reduction in the Petroleum Refining Industry. Ind. Eng. Chem. Res., 47:760-776. 
Escudero, L.; Quintana, F.; Salmerón, J. (1999). CORO, a modeling and an algorithmic framework for oil supply, transformation and distribution optimization under uncertainty. European Journal of Operational Research, 114(3): 638-656.

Franca, P.M.; Luna, H.P.L. (1982). Solving stochastic transportation-location problems by generalized benders decomposition. Transportation Science, 16(2):113-126.

Geoffrion, A.M.; Graves, G.W. (1974). Multicommodity distribution system design by benders decomposition. Management science, pages 822-844.

Grosmann, I. E.; Heever, S. A.; Harjunkoski, I. (2001). Discrete optimization methods and their role in the integration of planning and scheduling. Proceedings of Chemical Process Control Conference 6, Tucson.

Heitsch and Romisch, W. (2005). Scenario tree modelling for multistage stochastic programs. Preprint 296, DFG Research Center Matheon (Mathematics for key technologies), Berlin, Germany, (www.matheon.de).

Herroelen, W.; Leus, R. (2005). Project scheduling under uncertainty: Survey and research potentials. European Journal of Operational Research.

Higle, J.L.; Sen, S. (1999). An introductory tutorial on stochastic linear programming models. Interfaces 29:33-61.

Hussain, R.; Assavapokee, T.; Khumawala, B. (2006). Supply Chain Management in the Petroleum Industry: Challenges and Opportunities. International Journal of Global Logistics \& Supply Chain Management, v.1, n.2, p.90 - 97.

Jia, Z.; Ierapetritou, M. (2003). Mixed-Integer Linear Programming Model for Gasoline Blending and Distribution Scheduling. Industrial and Engineering Chemistry Research, v. 42 , p. $825-835$.

Joly, M.; Moro, L.F.L.; Pinto, J. (2002). Planning and scheduling for petroleum refineries using mathematical programming. Brazilian Journal of Chemical Engineering, v.19, n.2.

Kallrath, J. (2005). Solving planning and design problems in the process industry using mixed integer and global optimization. Annals of Operations Research, v. 140, n. 1, p. 339-373. 
Kleywegt, A.J.; Shapiro, A.; Homem-de Mello, T. (2002). The sample average approximation method for stochastic discrete optimization. SIAM Journal on Optimization, 12(2):479-502.

Lababidi, H.; Ahmed, M.; Alatiqi, I.; Al-Enzi, A. (2004). Optimizing the supply chain of a petrochemical company under uncertain operating and economic conditions. Industrial \& Engineering Chemistry Research, 43(1): 63-73.

Leiras, A.; Ribas, G.; Hamacher, S.; Elkamel, A. (2011). Literature Review of Oil Refineries Planning under Uncertainty. International Journal of Oil, Gas and Coal Technology, 4, 2,156 - 173.

Li, W.; Hui, C.; Li, P.; Li, A. (2004). Refinery Planning under Uncertainty. Industrial and Engineering Chemistry Research 43, 6742-6755.

Linderoth, J.; Wright, S. (2003). Decomposition algorithms for stochastic programming on a computational grid. Computational Optimization and Applications, 24(2):207-250.

Magnanti, T.L.; Mireault, P.; Wong, R.T. (1986). Tailoring benders decomposition for uncapacitated network design. Netow at Pisa, pages 112-154.

Manzano, F.S. (2005). Supply Chain Practices in the Petroleum Downstream. Engineering Systems Massachusetts Institute of Technology, Massachusetts. Mestrado.

Más, R.; Pinto, J. (2003). A Mixed-Integer Optimization Strategy for Oil Supply in Distribution Complexes. Optimization and Engineering, v.4, p.23 - 64.

Mitra, S. (2006). Scenario generation for stochastic programming. White paper, Optirisk Systems, UK, 1-34.

Nascimento, L. M. (2011). Planejamento da Produção de uma Refinaria sob Condições de Incerteza. Programa de Pós-graduação em Engenharia de Produção da COPPE, UFRJ, Rio de Janeiro. Mestrado.

Neiro, S.; Pinto, J. (2003). Supply Chain Optimization of Petroleum Refinery Complexes. FOCAPO, Coral Springs.

Neiro, S.; Pinto, J. (2004). A general modeling framework for the operational planning of petroleum supply chains. Computers and Chemical Engineering, v.28, n.6-7, p.871896. 
Neiro, S.; Pinto, J. (2005). Multiperiod Optimization for Production Planning of Petroleum Refineries. Chemical Engineering Communications, 192, 1, 62-88.

Neiro, S.; Pinto, J. (2006). Langrangean decomposition applied to multiperiod planning of petroleum refineries under uncertainty. Latin American Applied Research, 36, 4.

Pagnoncelli, B. K.; Bortolossi, H. J. (2008). Introdução à Otimização sob Incerteza. XI Simpósio de Pesquisa Operacional e Logística da Marinha, Rio de Janeiro.

Peng, X.; Jirutitijaroen, P. (2010). Convergence acceleration techniques for the stochastic unit commitment problem. Probabilistic Methods Applied to Power Systems (PMAPS), IEEE 11th International Conference, pages 364-371.

Pinto, J.; Moro, L. (2000). A planning model for petroleum refineries. Brazilian Journal of Chemical Engineering 17, 575-586.

Pongsakdia, A.; Rangsunvigit, P.; Siemanond, K.; Bagajewicz, M.J. (2006). Financial risk management in the planning of refinery operations. International Journal of Production Economics, 103, 64-86.

Ribas, G.P; Hamacher, S.; Oliveira, F. (2008). Um modelo para o planejamento estratégico da cadeia de petróleo. XL SBPO - Simpósio Brasileiro de Pesquisa Operacional, João Pessoa.

Saharidis, G.K.D.; Boile, M.; Theofanis, S. (2011). Initialization of the benders master problem using valid inequalities applied to fixed-charge network problems. Expert Systems with Applications, 38(6):6627-6636.

Santoso, T.; Ahmed, S.; Goetschalckx, M.; Shapiro, A. (2005). A stochastic programming approach for supply chain network design under uncertainty. European Journal of Operational Research, v.167, p.96 - 115.

Senne, L.F.F. (2009). Incorporação de incertezas no plano de abastecimento de uma empresa da indústria do petróleo. Programa de Pós-graduação em Engenharia de Produção da COPPE, UFRJ, Rio de Janeiro. Mestrado.

Slyke, R.M.V.; Wets, R. (1969). L-Shaped linear programs with applications to optimal control and stochastic programming. Society for Industrial and Applied Mathematics Journal on Applied Mathematics, 17(4): 638-663. 
Schütz, P.; Tomasgard, A.; Ahmed, S. (2009). Supply chain design under uncertainty using sample average approximation and dual decomposition. European Journal of Operational Research, 199(2):409-419.

Uster, H.; Agrahari, H. (2011). A benders decomposition approach for a distribution network design problem with consolidation and capacity considerations. Operations Research Letters.

Verderame, P. M.; Elia, J. A.; Li, J.; Floudas, C.A. (2010). Planning and scheduling under uncertainty: A review across multiple sectors. Ind. Eng. Chem. Res., 49, 39934017.

Verweij, B.; Ahmed, S.; Kleywegt, A.J.; Nemhauser, G.; Shapiro, A. (2003). The sample average approximation method applied to stochastic routing problems: a computational study. Computational Optimization and Applications, 24(2):289-333.

Yang, Y.; Lee, J.M. (2011). Acceleration of benders decomposition for mixed integer linear programming. Advanced Control of Industrial Processes (ADCONIP), International Symposium, pages 222-227. 